\title{
Robust Tracking Enhancement of Robot Systems Including Motor Dynamics: A Fuzzy-Based Dynamic Game Approach
}

\author{
Bor-Sen Chen, Senior Member, IEEE, Huey-Jian Uang, and Chung-Shi Tseng
}

\begin{abstract}
A robust tracking control design of robot systems including motor dynamics with parameter perturbation and external disturbance is proposed in this study via adaptive fuzzy cancellation technique. A minimax controller equipped with a fuzzy-based scheme is used to enhance the tracking performance in spite of system uncertainties and external disturbance. The design procedure is divided into three steps. At first, a linear nominal robotic control design is obtained via model reference tracking with desired eigenvalue assignment. Next, a fuzzy logic system is constructed and then tuned to eliminate the nonlinear uncertainties as possibly as it can to enhance the tracking robustness. Finally, a minimax control scheme is specified to optimally attenuate the worst-case effect of both the residue due to fuzzy cancellation and external disturbance to achieve a minimax tracking performance. In addition, an adaptive fuzzybased dynamic game theory is introduced to solve the minimax tracking problem. The proposed method is appropriate for the robust tracking design of robotic systems with large parameter perturbation and external disturbance. A simulation example of a two-link robotic manipulator driven by de motors is also given to demonstrate the effectiveness of proposed design method's tracking performance.
\end{abstract}

Index Terms - Fuzzy-based dynamic game theory, fuzzy cancellation, tracking enhancement, uncertain robot systems.

\section{INTRODUCTION}

$\mathbf{I}$ $\mathrm{N}$ robotics literature, a robotic dynamic model is always described by a second-order differential equation at each joint of arm with the acting torque (or force) as the control input reference. Extensive approaches had been proposed for the feedback control of robot arms [1]-[4], [7]-[8]. A total robot model (arm dynamics plus motor (actuator) dynamics and the interaction between motors and joints) have recently been considered in robotic control design [1], [3], [5], [6]. Under this circumstance, a third-order robot dynamic model must be developed to include the actuator dynamics. Owing to factors such as high-velocity moment, highly varying loads, friction, and saturation of actuator, the robotic system becomes uncertain. In [5], a robust control is developed on the basis of the robust stabilization technique in [16], [17]. In this

Manuscript received October 7, 1996; revised March 27, 1998. This work was supported by the National Science Council under Contract NSC 86-2213E-007-031.

The authors are with the Control and Signal Processing Laboratory, Department of Electrical Engineering, National Tsing Hua University, Hsin Chu, Taiwan, 30043 R.O.C.

Publisher Item Identifier S 1063-6706(98)08253-8. approach, the nonlinear uncertain dynamics are assumed to be norm bounded. A robust controller based on a small gain theorem is then derived to guarantee the global boundedness of robotic system. The result may be conservative if the plant perturbations are large. Furthermore, if only stability is guaranteed, no further tracking performance is achieved.

$H_{\infty}$ attenuation control design has been widely studied in the recent decade for desired disturbance attenuation [12]-[14]. An $H_{\infty}$ tracking control design has been developed for robotic systems to attenuate the worst-case effect of parameter perturbations and external noises [15]. However, if the magnitudes of uncertain dynamics and external noises are large, the effect on tracking error may still be obvious or even lead to system instability. In this situation, before an $H_{\infty}$ attenuation is applied, effectively eliminating uncertain dynamics is an appealing strategy for enhancing tracking performance.

Conventionally, adaptive control schemes can be employed to match this uncertain nonlinear dynamics. However, linear parametrization must be assumed in conventional adaptive control schemes, i.e., the unknown parameters must be of linear structure. In nonlinear uncertain robotic systems this may not be true. Since fuzzy logic system can be tuned to approximate any nonlinear dynamic with model free, in this paper, a fuzzy scheme is used to efficiently eliminate the plant uncertainties so that tracking performance can be enhanced via adaptive learning method.

Fuzzy techniques have been recently used to effectively approximate unknown nonlinear dynamics [9]-[11]. However, in the conventional adaptive fuzzy control, the desired tracking performance cannot be guaranteed from the more theoretical control perspective. More recently [26], an $H_{\infty}$ adaptive tracking control has been proposed for single-input singleoutput (SISO) unknown nonlinear systems via an adaptive fuzzy control based on feedback linearization technique and $H_{\infty}$ control scheme. However, the limitation of this method is that it employs feedback linearization-based adaptive fuzzy control, which contains an inverse term of adaptive fuzzy logic system. Therefore, it is sensitive, particularly, in the case of approaching a small value. Furthermore, only SISO systems have been discussed, but robotic tracking systems are multi-input multi-output (MIMO) nonlinear systems. Unlike conventional fuzzy control schemes for the nonlinear robotic system, the proposed adaptive scheme is used to cancel the nonlinear part of robotic system so that the robotic system 
becomes a linear system with a cancellation residue. Then a linear $H_{\infty}$ control scheme is employed to achieve the robust tracking. Recently, a neural-based adaptive $H_{\infty}$ control has been applied to robotic systems via adaptive feedback linearization method in [28].

Unlike the adaptive control design method, in this study, a linear nominal robotic system is given and only robust tracking problem for uncertain dynamics and external disturbance is addressed. A fuzzy-based minimax scheme is employed to cancel the effects of nonlinear uncertainties to enhance the robust tracking of uncertain robotic systems. The conventional adaptive feedback linearization technique for uncertain nonlinear system control design is unnecessary in this work.

The proposed controller contains two terms, one contains a self-tuning fuzzy logic system which is developed to optimally cancel the uncertain dynamics to enhance the tracking robustness, the other contains a minimax control algorithm that is employed to optimally attenuate the worst-case effect of both the residue due to adaptive fuzzy cancellation and the exogenous disturbance below a desired level from a minimax perspective.

The proposed design method attempts to combine the dynamic game technique and adaptive fuzzy cancellation algorithm to guarantee a robust tracking performance for uncertain robotic control systems including motor dynamics. In the proposed robust tracking control, the adaptive fuzzy cancellation technique plays a role of rough tuning and the minimax attenuation technique plays a role of fine tuning. Unlike the conventional adaptive control in robotic systems, the uncertain dynamics do not need to have a linear parameterized structure in this study, i.e., uncertain dynamics can be free of structure in the proposed fuzzy-based minimax control systems. Since the adaptive feedback linearization technique is avoided, an inverse adaptive matrix is unnecessary. Hence, the computational complexity and sensitivity of control algorithm are significantly reduced. Therefore, the proposed design method is appropriate for the robust tracking control design of robotic systems with large uncertainties and external disturbances.

In this paper, Section II presents a dynamic model of the robotic manipulator and joint motors first. The system has a state vector composed of the errors in joint position, velocity and acceleration and a control vector composed of the armatures' input voltages. In Section III, the robust minimax tracking problem for uncertain robotic system via fuzzy-based control scheme is described. The uncertainty is matched and canceled by an adaptive fuzzy scheme. In this approach, we propose a fuzzy-based dynamic game scheme to treat the nonlinear robust tracking control design of robotic manipulators, including actuator dynamics. The proposed scheme is simpler and more robust than the approaches proposed in [3]-[6]. In Section IV, the proof of the minimax tracking problem via adaptive fuzzy cancellation scheme is given and the design procedures is proposed. In Section $\mathrm{V}$, a simulation example is provided to demonstrate the design procedure's effectiveness and to confirm the performance of the proposed robust tracking control design for robotic systems with consideration of motor drives.
Simulation results indicate that a desired robust tracking performance can be guaranteed for an uncertain robotic system via the proposed fuzzy cancellation-based minimax algorithm under large time-varying parameter perturbations and external disturbance. Concluding remarks are finally made in Section VI.

\section{CONTROL Dynamics OF ROBOT AND JoInt Motors}

A dynamic model of robotic manipulator and joint motors is first described. Following Tarn et al. [3], Beekman and Lee [6], and Mahmoud [5], the joints of robotic manipulators driven by $\mathrm{dc}$ motor are considered and a combined dynamic model of the robot manipulator plus joint motors is developed herein. An $n$-joints robot dynamic (including actuators) is described as follows. First, the motion equations of a robotic manipulator with revolute joints can be expressed as

$$
M^{\prime}(q) \ddot{q}+C(q, \dot{q}) \dot{q}+G(q)=\tau+d^{\prime}
$$

where the following notations apply:

$q, \dot{q}, \ddot{q} \in R^{n}$ vectors of joint positions, velocities, and accelerations;

$M^{\prime}(q) \in R^{n \times n}$ matrix of the moment inertia;

$C(q, \dot{q}) \dot{q} \in R^{n}$ vector of the centripetal and Coriolis forces;

$G(q) \in R^{n}$ vector of gravitational force;

$\tau \in R^{n}$ vector of torques developed at the joint side of gear box;

$d^{\prime} \in R^{n}$ external disturbance.

Remark: The matrix $M^{\prime}$ in the robot model (1) is symmetric positive-definite.

The relation between the joint position $q$ and the motor-shaft position $q_{m}$ is given by

$$
q_{m}=N q
$$

where $N \in R^{n \times n}$ is a diagonal matrix of the gear ratios for the $n$ joints and $N>0$ (which means that the matrix $N$ is positive-definite). By armature-controlled dc motors, the electrical model of the $j$ th motor is characterized by

$$
R_{j} i_{j}+L_{j} \frac{d i_{j}}{d t}+K_{b_{j}} \frac{d q_{m_{j}}}{d t}=u_{j}, \quad \text { for } j=1,2, \cdots n \text {. }
$$

where $R_{j}$ is the resistance of the armature circuit, $L_{j}$ is the inductance of the armature circuit, $K_{b j}$ is the back electromotive force (EMF) constant of the motor, $i_{j}$ is the armature current, $q_{m_{j}}$ is the motor shaft position, and $u_{j}$ is the armature input voltage. Let us define

$$
\begin{gathered}
R=\operatorname{diag}\left[R_{j}\right], \quad L=\operatorname{diag}\left[L_{j}\right], \quad K_{b}=\operatorname{diag}\left[K_{b_{j}}\right] \\
u=\left[\begin{array}{c}
u_{1} \\
u_{2} \\
\vdots \\
u_{n}
\end{array}\right], \quad i=\left[\begin{array}{c}
i_{1} \\
i_{2} \\
\vdots \\
i_{n}
\end{array}\right], \quad q_{m}=\left[\begin{array}{c}
q_{m_{1}} \\
q_{m_{2}} \\
\vdots \\
q_{m_{n}}
\end{array}\right]
\end{gathered}
$$

where diag[·] denotes a diagonal matrix of dimension $n$. Then, the electrical models of armature-controlled dc motors in (3) 
can be grouped in a compact form

$$
R i+L \frac{d i}{d t}+K_{b} \dot{q}_{m}=u .
$$

Furthermore, the developed torque at the joint side is related to the armature current by

$$
\tau=N K_{t} i
$$

where $K_{t} \in R^{n \times n}$ is the diagonal matrix of motor torque constants and $K_{t}>0$. Substituting (7) into (6) yields

$$
R_{n} \tau+L_{n} \dot{\tau}+K_{b n} \dot{q}=u
$$

where

$$
R_{n}=R\left(N K_{t}\right)^{-1}, \quad L_{n}=L\left(N K_{t}\right)^{-1}, \quad K_{b n}=K_{b} N
$$

Remark: The indicated inverse in (9) exists due to the physical nature of $N$ and $K_{t}$.

Now, to obtain the combined robot dynamic model, we substitute (1) into (8). Thus

$$
\begin{aligned}
& L_{n} M^{\prime}(q) q^{(3)}+\left(R_{n} M^{\prime}(q)+L_{n}\left[\dot{M}^{\prime}(q)+C(q, \dot{q})\right]\right) \ddot{q} \\
& \quad+\left(R_{n} C(q, \dot{q})+L_{n} \dot{C}(q, \dot{q})+K_{b n}\right) \dot{q} \\
& \quad+R_{n} G(q)+L_{n} \dot{G}(q)=u+R_{n} d^{\prime}+L_{n} \dot{d}^{\prime}
\end{aligned}
$$

where $q^{(3)}$ denotes the third partial time derivative of $q$. The dynamic model in (10) can be written in the following compact form:

$$
M(q) q^{(3)}+D(q, \dot{q}, \ddot{q})=u+R_{n} d^{\prime}+L_{n} \dot{d}^{\prime}
$$

where

$$
\begin{aligned}
M(q)= & L_{n} M^{\prime}(q) \\
D(q, \dot{q}, \ddot{q})= & \left(R_{n} M^{\prime}(q)+L_{n}\left[\dot{M}^{\prime}(q)+C(q, \dot{q})\right]\right) \ddot{q} \\
& +\left(R_{n} C(q, \dot{q})+L_{n} \dot{C}(q, \dot{q})+K_{b n}\right) \dot{q} \\
& +R_{n} G(q)+L_{n} \dot{G}(q) .
\end{aligned}
$$

By introducing the state vector

$$
x=\left[\begin{array}{l}
x_{1} \\
x_{2} \\
x_{3}
\end{array}\right] \triangleq\left[\begin{array}{l}
q \\
\dot{q} \\
\ddot{q}
\end{array}\right]
$$

Equation (11) is transferred into the following standard form:

$$
\begin{aligned}
\dot{x}= & {\left[\begin{array}{c}
x_{2} \\
x_{3} \\
-M^{-1}\left(x_{1}\right) D(x)
\end{array}\right]+\left[\begin{array}{c}
0 \\
0 \\
M^{-1}\left(x_{1}\right)
\end{array}\right] u } \\
& +\left[\begin{array}{c}
0 \\
0 \\
M^{-1}\left(x_{1}\right)\left(R_{n} d^{\prime}+L_{n} \dot{d}^{\prime}\right)
\end{array}\right] .
\end{aligned}
$$

Here we denote $d=M^{-1}\left(x_{1}\right)\left(R_{n} d^{\prime}+L_{n} \dot{d}^{\prime}\right)$ and assume $d \in L_{2}\left[0, t_{f}\right]$. Therefore, (15) can be rewritten as

$$
\begin{aligned}
\dot{x}= & {\left[\begin{array}{c}
x_{2} \\
x_{3} \\
-M^{-1}\left(x_{1}\right) D(x)
\end{array}\right]+\left[\begin{array}{c}
0 \\
0 \\
M^{-1}\left(x_{1}\right)
\end{array}\right] u } \\
& +\left[\begin{array}{l}
0 \\
0 \\
I
\end{array}\right] d
\end{aligned}
$$

which represents a typical nonlinear dynamical system with an affine nonlinear input function. In this study, we assume the state vector $x$ can be measured.

The state vector in (16) is composed of joint positions, velocities and accelerations, and the input vector $u$ consists of the armatures' input voltages. Because of the model's complexity and nonlinearity in (16), directly designing control laws is not easy. This situation is further compounded by the drift incurred in on-line measurements of acceleration, the frequent changes in load and model parameter, and the corruption of external disturbances.

Given a task of a continuously differentiable and uniformly bounded trajectory in the joint space $q_{d}$ for which we wish the robot manipulator to follow. Therefore, we define

$$
\tilde{q}=q-q_{d}=x_{1}-q_{d}
$$

as the joint position error. Tarn et al. developed a feedback linearization plus decoupling technique based on differential geometric control theory to provide a nonlinear feedback control law for the regulation of robotic arms [3]. However, this design is possible only while the dynamics of the robotic dynamic are well known. Assume that $M_{0}(\cdot)$ and $D_{0}(\cdot, \cdot, \cdot)$ are the nominal estimates of $M(\cdot)$ and $D(\cdot, \cdot, \cdot)$, respectively. We follow the notion in [5] and use the following control law:

$$
u=M_{0}\left(q_{d}^{(3)}-K_{1} \tilde{q}-K_{2} \dot{\tilde{q}}-K_{3} \ddot{\tilde{q}}+u_{0}\right)+D_{0}(q, \dot{q}, \ddot{q})
$$

where $K_{1}, K_{2}, K_{3}$ are diagonal matrices to be designed and $u_{0}$ is an auxiliary control signal yet to be specified.

Substituting (17) and (18) into (16) leads to

$$
\dot{x}=\left[\begin{array}{c}
x_{2} \\
x_{3} \\
M^{-1}\left(D_{0}-D\right)+M^{-1} M_{0} v+d
\end{array}\right]
$$

where

$$
v=q_{d}^{(3)}-K_{1} \tilde{q}-K_{2} \dot{\tilde{q}}-K_{3} \ddot{\tilde{q}}+u_{0} .
$$

For further development, let us define the system uncertainties as

$$
f(x)=M^{-1}\left[\left(D_{0}-D\right)+\left(M_{0}-M\right) v\right] .
$$

For design purpose, let us denote

$$
e=\left[\begin{array}{c}
\tilde{q} \\
\stackrel{\tilde{q}}{\ddot{q}}
\end{array}\right]=\left[\begin{array}{l}
x_{1}-q_{d} \\
x_{2}-\dot{q}_{d} \\
x_{3}-\ddot{q}_{d}
\end{array}\right]
$$




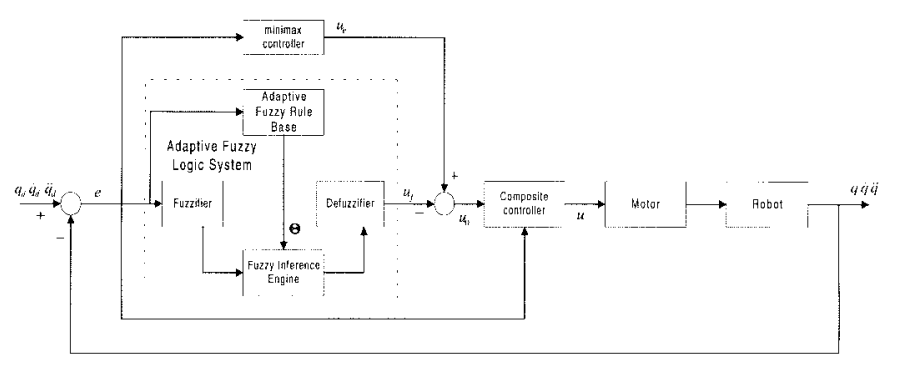

Fig. 1. Fuzzy logic system for robot tracking control.

as the state error vector. From (21), if our nominal estimates of $M$ and $D$ are exact (i.e., $M_{0}=M$ and $D_{0}=D$ ) then the system uncertainties will be zero except the external noise. This is impossible in practical robot systems. In order to enhance the robust tracking performance, an adaptive fuzzy logic system (Fig. 1) is introduced herein to eliminate the uncertainties.

In the form of (20) and (21), we obtain a simple form of (19)

$$
\dot{x}=\left[\begin{array}{c}
x_{2} \\
x_{3} \\
v+f(x)+d(t)
\end{array}\right]
$$

From (17), (19), and (22), we obtain

$$
\begin{aligned}
& {\left[\begin{array}{c}
\dot{\tilde{q}} \\
\stackrel{\tilde{q}}{\tilde{q}^{(3)}}
\end{array}\right]=\left[\begin{array}{c}
\dot{\tilde{q}} \\
\stackrel{\tilde{\tilde{q}}}{\ddot{\tilde{q}}}+u_{0}+f(x)+d
\end{array}\right]} \\
& =\left[\begin{array}{ccc}
0_{n \times n} & I_{n \times n} & 0_{n \times n} \\
0_{n \times n} & 0_{n \times n} & I_{n \times n} \\
-K_{1} & -K_{2} & -K_{3}
\end{array}\right]\left[\begin{array}{c}
\tilde{q} \\
\stackrel{\tilde{q}}{\tilde{q}}
\end{array}\right] \\
& +\left[\begin{array}{c}
0_{n \times n} \\
0_{n \times n} \\
I_{n \times n}
\end{array}\right] u_{0} \\
& +\left[\begin{array}{c}
0_{n \times n} \\
0_{n \times n} \\
I_{n \times n}
\end{array}\right](f(x)+d) \text {. }
\end{aligned}
$$

The system in (24) represents the robotic tracking error dynamics. In terms of the terminology of Garofalo and Leitmann [18], it is a nominally linear uncertain system. The nonlinear uncertainties are modeled by the vector $f(x)$. A more convenient form of (24) would be

$$
\dot{e}=A e+B u_{0}+B f(x)+B d(t)
$$

where

$$
A=\left[\begin{array}{ccc}
0 & I & 0 \\
0 & 0 & I \\
-K_{1} & -K_{2} & -K_{3}
\end{array}\right], \quad B=\left[\begin{array}{l}
0 \\
0 \\
I
\end{array}\right]
$$

Remark: The control parameters $K_{1}, K_{2}$, and $K_{3}$ are specified so that $A$ has desired eigenvalues and the tracking dynamic in (25) has a desired response while the robotic system is free of uncertainty $f(x)$ and external disturbance $d(t)$.

\section{PROBlem Formulation}

The tracking error dynamics of uncertain robotic manipulator with actuator dynamics are described by (25) in the above section. If the robotic system is free of uncertainties and external disturbance (i.e., $f(x)=0$ and $d(t)=0$ ), by the nonlinear control law in (18) with adequate choice of linear control $u_{0}(t)$, the tracking error $e(t)$ in (25) will asymptotically converge to zero as $t \rightarrow \infty$ [2]. In practical robotic systems, however, uncertainties due to parameter perturbations, unmodeled dynamics, and external noises are inevitable. These uncertainties deteriorate the tracking performance or even lead to system instability in the worst case. Hence, the effect of uncertainties $f(x)$ and $d(t)$ on tracking error in (25) must be eliminated. Since is uncertain, several robust design algorithms [16]-[18] have been employed as a robust controller to override the upper norm bound of $\|f(x)\|$. Due to high nonlinearity and uncertainty of $f(x)$, estimating its upper norm bound is a difficult task. Furthermore, effectively suppressing the effect of the external noise $d(t)$ is not easy, for example, by variable structure system (VSS) control or dead-zone control based on an upper bound of external disturbance to treat this problem. All of these robust control methods may lead to conservative and imprecise result.

In this paper, the control signal $u_{0}$ is divided into two parts as follows:

$$
u_{0}=u_{e}-u_{f}(x, \Theta)
$$

Under this circumstance, the tracking error dynamics in (25) is of the following form:

$$
\dot{e}=A e+B u_{e}+B\left(f(x)-u_{f}(x, \Theta)\right)+B d
$$

where $u_{f}(x, \Theta)$ is a fuzzy logic system and $\Theta(t)$ is a parameter matrix to be tuned.

In (28), the fuzzy logic system $u_{f}(x, \Theta)$ will be tuned to approximate $f(x)$ as closely as possible. Furthermore, the control signal $u_{e}(t)$ will be used to attenuate the total effect of the residue of $f(x)-u_{f}(x, \Theta)$ and external disturbance $d(t)$ on the tracking error $e(t)$ from a minimax perspective. In this paper, since the fuzzy logic system $u_{f}(x, \Theta)$ is employed to cancel the uncertain $f(x)$ in (28) to enhance the robust tracking, the fuzzy logic system $u_{f}(x, \Theta)$ in Fig. 1 is described in the following paragraphs.

The fuzzy logic systems can perform universal approximation from the perspective of human experts and can uniformly approximate nonlinear continuous functions to arbitrary accuracy [19], [20]. The fuzzy logic systems in Fig. 1 are qualified as building blocks of $u_{f}(x, \Theta)$ for adaptive cancellation of nonlinear function $f(x)$ in (28). The fuzzy logic systems are constructed from the fuzzy If-Then rules using some specific inference, fuzzification, and defuzzification strategies. Therefore, linguistic information from human experts and the information from state measurement can be incorporated into fuzzy logic systems to adjust their parameters to achieve optimal approximation [25]. 
The basic configuration of a fuzzy logic system depicts in Fig. 1 as a fuzzy logic system. The fuzzy logic system $u_{f}(x, \Theta)$ in this work performs a mapping from $U \in R^{3 n}$ to $V \in R^{n}$. Let $U=U_{1} \times U_{2} \times U_{3}$, where $U_{i} \in R^{n}$, for $i=1,2,3$. A fuzzy rule base consists of a collection of fuzzy If-Then rules [19], [20], [25]

$$
\begin{aligned}
R^{(l)}: & \text { If } x_{1} \text { is } F_{1}^{l} \text {, and } \cdots \text { and, } x_{3 n} \text { is } F_{3 n}^{l} \\
& \text { Then } u_{f} \text { is } G^{l}
\end{aligned}
$$

where $x=\left(x_{1}, x_{2}, \cdots, x_{3 n}\right)^{T} \in U$ and $u_{f} \in V \subset R^{n}$ are the input and output of the fuzzy logic system, respectively, and $l=1,2, \cdots, M$. The fuzzy inference engine performs a mapping from fuzzy sets in $U \in R^{3 n}$ to fuzzy sets in $R^{3 n}$ based upon the fuzzy If-Then rules in the fuzzy rule base and the compositional rule of inference. The fuzzifier maps a crisp point $x=\left(x_{1}, x_{2}, \cdots, x_{3 n}\right)^{T} \in U$ into a fuzzy set $A_{x}$ in $U$. The defuzzifier maps a fuzzy set in $U$ to a crisp point in $V$. More information can be found in [19], [20], [25].

The fuzzy logic systems of Fig. 1 comprise an extremely rich class of static systems mapping from $U \subset R^{3 n}$ to $V \subset R^{n}$ because many different choices are available within each block. In addition, many combinations of these choices can result in a useful subclass of fuzzy logic systems. One subclass of fuzzy logic systems is used here as building blocks of our adaptive fuzzy approximation (cancellation) controller and is described by the following important result.

Lemma [25]: The fuzzy logic systems with center-average defuzzifier, product inference and singleton fuzzifier are in the following form:

$$
u_{f_{i}}(x, \theta)=\frac{\sum_{l=1}^{M} \theta_{i l}\left(\prod_{j=1}^{3 n} \mu_{F_{j}^{l}}\left(x_{i}\right)\right)}{\sum_{l=1}^{M}\left(\prod_{j=1}^{3 n} \mu_{F_{j}^{l}}\left(x_{j}\right)\right)}
$$

where $\theta_{i l}$ is the point at which the given membership function $\mu_{F_{j}^{l}}\left(x_{j}\right)$ achieves its maximum value, and we assume that $\mu_{F_{j}^{l}}\left(\theta_{i l}\right)=1$.

Let us denote the fuzzy basis functions as

$$
\varsigma_{l}(x)=\frac{\prod_{j=1}^{3 n} \mu_{F_{j}^{l}}\left(x_{i}\right)}{\sum_{l=1}^{M}\left(\prod_{j=1}^{3 n} \mu_{F_{j}^{l}}\left(x_{j}\right)\right)}
$$

for $l=1,2, \cdots, M$ and denote

$$
\varsigma(x) \triangleq\left[\varsigma_{1}(x) \quad \varsigma_{2}(x) \quad \cdots \quad \varsigma_{M}(x)\right] .
$$

Consequently, $u_{f_{i}}(x, \Theta)$ in (30) is of the following form:

$$
\begin{aligned}
& u_{f_{i}}(x, \Theta)=\left[\begin{array}{llll}
\varsigma_{1}(x) & \varsigma_{2}(x) & \cdots & \varsigma_{M}(x)
\end{array}\right] \\
& \times\left[\begin{array}{c}
\theta_{i_{1}}(t) \\
\theta_{i_{2}}(t) \\
\vdots \\
\theta_{i_{M}}(t)
\end{array}\right] \\
& \triangleq \varsigma(x) \theta_{i}(t)
\end{aligned}
$$

where $\theta_{i}(t)=\left[\begin{array}{llll}\theta_{i_{1}}(t) & \theta_{i_{2}}(t) & \cdots & \theta_{i_{M}}(t)\end{array}\right]^{T}$ for $i=$ $1,2, \cdots n$. Therefore, the fuzzy logic system for the $n$ input $-n$ output of $n$-link robotic system with the same fuzzy basis functions is of the following form:

$$
\begin{aligned}
u_{f}(x, \Theta)= & {\left[\begin{array}{c}
u_{f_{1}}(x, \theta) \\
u_{f_{2}}(x, \theta) \\
\vdots \\
u_{f_{n}}(x, \theta)
\end{array}\right]=\left[\begin{array}{c}
\varsigma(x) \theta_{1}(t) \\
\varsigma(x) \theta_{2}(t) \\
\vdots \\
\varsigma(x) \theta_{n}(t)
\end{array}\right] } \\
= & {\left[\begin{array}{cccc}
\varsigma(x) & 0 & \cdots & 0 \\
0 & \varsigma(x) & \ddots & \vdots \\
\vdots & \ddots & \ddots & \vdots \\
0 & \cdots & 0 & \varsigma(x)
\end{array}\right] } \\
& \times\left[\begin{array}{c}
\theta_{1}(t) \\
\theta_{2}(t) \\
\vdots \\
\theta_{n}(t)
\end{array}\right]
\end{aligned}
$$

i.e.,

$$
u_{f}(x, \Theta)=\xi(x) \Theta(t)
$$

where

$$
\begin{aligned}
\xi(x) & =\left[\begin{array}{cccc}
\varsigma(x) & 0 & \cdots & 0 \\
0 & \varsigma(x) & \ddots & \vdots \\
\vdots & \ddots & \ddots & \vdots \\
0 & \cdots & 0 & \varsigma(x)
\end{array}\right] \\
\Theta(t) & =\left[\begin{array}{c}
\theta_{1}(t) \\
\theta_{2}(t) \\
\vdots \\
\theta_{n}(t)
\end{array}\right] .
\end{aligned}
$$

Remarks:

1) The membership function $\mu_{F_{i}^{l}}$ can be triangular or any type of membership functions.

2) In general, as the number $M$ of fuzzy basis functions approaches infinite [25], fuzzy logic system $u_{f}(x, \Theta)$ can approximate any uncertain function $f(x)$ by adequately selecting parameter matrix $\Theta$ in (35).

3) In this design, the membership functions are specified by the designer's experience or knowledge regarding the uncertainties of a robotic system and the parameter $\Theta$ is to be tuned according to the tracking error $e(t)$. 
In practical control design, for the convenience of computation and implementation, the number of fuzzy basis functions of fuzzy logic system is chosen as small as possible. Under this circumstance, an adaptive law must be developed to tune the parameter $\Theta(t)$ to construct a fuzzy logic system $u_{f}(x, \Theta)$ with adequate dimension approximate to $f(x)$ as closely as possible.

Now define the following optimal approximation [25]:

$$
\Theta^{*}=\arg \min _{\Theta \in \Omega} \max _{x \in \Omega_{x}}\|f(x)-\xi \Theta\|
$$

where $\|\cdot\|$ denotes the Euclidean norm, i.e., $\|x\|=\sqrt{x^{T} x} . \Omega$ and $\Omega_{x}$ denote the sets of suitable bounds on $\Theta(t)$ and $x$, respectively. We assume that $\Theta(t)$ and $x$ never reach the boundary of $\Omega$ and $\Omega_{x}$ and $f(x)=\xi \Theta^{*}+\varepsilon(t), \varepsilon(t) \leq \bar{\varepsilon}$ in $\Omega$ and $\Omega_{x}$, for some $\bar{\varepsilon}>0$. Otherwise, the projection algorithm such as described in the remark following the proof of Theorem must be introduced to prevent the divergence of $\Theta(t)$.

Consequently, the tracking error dynamic (28) can be rewritten as follows:

$$
\begin{aligned}
\dot{e}= & A e+B u_{e}+B\left(\xi \Theta^{*}-\xi \Theta\right)+B\left(f(x)-\xi \Theta^{*}\right) \\
& +B d(t) \\
= & A e+B u_{e}+B \xi \tilde{\Theta}+B w(t)
\end{aligned}
$$

where $w(t) \triangleq f(x)-\xi \Theta^{*}+d(t)$ denotes the sum of the optimal cancellation error via fuzzy logic system and external disturbance and $\tilde{\Theta}(t)$ is defined as

$$
\tilde{\Theta}(t) \triangleq \Theta^{*}-\Theta(t) .
$$

Our design procedure is divided into two steps. In the first step, the adaptive fuzzy algorithm $u_{f}(x, \Theta)$ is tuned via $\Theta(t)$ to optimally cancel the uncertain term $f(x)$. Under such a circumstance, the term $\xi \Theta^{*}-\xi \Theta=\xi \tilde{\Theta}$ will finally vanish. If the effect of $w(t)$ can not be eliminated efficiently, the tracking performance will be deteriorated, particularly, in the case of a small number of membership functions in (32). This is a weak point to overcome in fuzzy-based control design methods. However, the cancellation error and external disturbance (i.e., $w(t))$ are uncertain and cannot be estimated perfectly and canceled completely. If such a situation arises, their worstcase effect on tracking error must be attenuated as fully as possible. Therefore, in the second step, the control signal $u_{e}(t)$ should be specified such that the worst-case effect of $w(t)$ on the tracking error $e(t)$ must be attenuated as much as possible and below a prescribed level $\rho$, i.e., the following minimax tracking performance must be satisfied for the tracking error dynamics in (37) (see [12], [13], [27])

$$
\begin{aligned}
& \min _{u_{e}(t) \in L_{2}\left[0, t_{f}\right]} \max _{w(t) \in L_{2}\left[0, t_{f}\right]} \int_{0}^{t_{f}}\left(e^{T}(t) Q e(t)+u_{e}^{T}(t) R u_{e}(t)\right. \\
& \left.-\rho^{2} w^{T}(t) w(t)\right) d t \\
& \leq e^{T}(0) P e(0)+\frac{1}{\gamma} \tilde{\Theta}^{T}(0) \tilde{\Theta}(0)
\end{aligned}
$$

where $\gamma>0$ is a weighting factor, $Q=Q^{T}>0, P=P^{T}>0$ and $R>0$ are some positive definite weighting matrices and the final time $t_{f}>0$ [12]-[15].
Our design objective involves how to tune the parameters of the fuzzy logic system to eliminate the term $\Theta^{*} \xi-\Theta \xi$ and then to specify an adequate minimax control law $u_{e}(t)$ in (37) so that the worst-case effect of $w(t)$ on $e(t)$ is guaranteed to be less than or equal to $\rho$. Under the case $e(0)=0$ and $\tilde{\Theta}(0)=0$, (39) can be rewritten as the following minimum $L_{2}$-gain problem:

$$
\begin{aligned}
& \min _{u_{e}(t) \in L_{2}\left[0, t_{f}\right]} \max _{w(t) \in L_{2}\left[0, t_{f}\right]} \frac{\left\|\left[Q^{1 / 2} e(t) R^{1 / 2} u_{e}(t)\right]\right\|_{L_{2}}}{\|w(t)\|_{L_{2}}} \\
& \leq \rho
\end{aligned}
$$

where $\|w(t)\|_{L_{2}} \triangleq \sqrt{\int_{0}^{t_{f}} w^{T}(t) w(t) d t}$ and

$$
\begin{aligned}
&\left\|\left[Q^{1 / 2} e(t) R^{1 / 2} u_{e}(t)\right]\right\|_{L_{2}} \\
& \triangleq \sqrt{\int_{0}^{t_{f}} e^{T}(t) Q e(t)+u_{e}^{T}(t) R u_{e}(t) d t} .
\end{aligned}
$$

However, in the case $e(0) \neq 0$ and $\tilde{\Theta}(0) \neq 0$, the minimax effect problem in (40) must be modified to (39) to consider $e(0)$ and $\tilde{\Theta}(0)$ as some kind of disturbance [12], [13].

At present [12]-[15], the minimax control (or the minimun $H_{\infty}$ control) is the most efficient method of eliminating the worst-case effect of the uncertain $w(t)$ on $e(t)$ in (37). Therefore, it will be employed to attenuate the effect of $w(t)$ to achieve the minimax robust tracking in our fuzzy-based control design of robotic system.

The expression in (40) implies that the induced $L_{2}$ norm from $w(t)$ to $Q^{1 / 2} e(t)$ and $R^{1 / 2} u_{e}(t)$ must be less than or equal to $\rho$. The physical meaning is that the worst-case influence of $w(t)$ on $e(t)$ and $u(t)$ must be less than or equal to $\rho$ for any $w(t) \in L_{2}\left[0, t_{f}\right]$ from an energy perspective. Owing to this reasoning, we are actually dealing with the minimax tracking problem for the model reference robotic control systems via an adaptive fuzzy cancellation scheme. The primary difference between the proposed algorithm and the conventional $H_{\infty}$ control is that an adaptive fuzzy control $u_{f}(x, \Theta)$ is employed in our design to eliminate the uncertain term $f(x)$ as much as possible to enhance the tracking robustness before minimax control $u_{e}(t)$ is used. In the conventional $H_{\infty}$ control design, only $H_{\infty}$ control $u_{e}(t)$ is employed to directly attenuate the uncertain term $f(x)$. Since $f(x)$ is generally very large, it may lead to system instability. Furthermore, its effect on tracking error will be still very large even when $H_{\infty}$ attenuation is used. The study in [15] is a case of $H_{\infty}$ tracking control design of robotic manipulator without employing the adaptive fuzzy cancellation scheme to eliminate the uncertainties. Therefore, the result is conservative and more control effort is deemed necessary. In our design, using an adaptive fuzzy logic system $u_{f}(x, \Theta)$ involves eliminating the uncertain term $f(x)$ as much as possible to enhance the tracking robustness. Employing $u_{e}(t)$ to attenuate the effect of $w(t)$ involves achieving a prescribed tracking performance. The role of adaptive fuzzy cancellation in the proposed robust tracking control design of uncertain 
robotic systems is discussed in the simulation example of Section V.

\section{Remarks:}

1) In general, the cancellation error and external disturbance $w(t)$ always exists; $\rho<1$ is necessary for the attenuation of $w(t)$ to achieve robust tracking. If $\rho \rightarrow$ $\infty$, then the robust minimax tracking performance design is reduced to a conventional $\mathrm{H}_{2}$ optimal tracking control design without considering the attenuation of $w(t)$ (see [12]-[15]). In this case, the tracking performance is deteriorated by $w(t)$ and is indicated in our simulation example in Section V.

2) The performance in (39) or (40) is of finite-time minimax (or $H_{\infty}$ ) tracking control so $w(t)$ can be finite in $\left[0, t_{f}\right]$. If $t_{f} \rightarrow \infty$, the integrations $\int_{0}^{\infty} e^{T}(t) Q e(t)+$ $u_{e}^{T}(t) R u_{e}(t) d t$ and $\int_{0}^{\infty} w^{T}(t) w(t) d t$ may increase to $\infty$. However, the integral inequality (40) always holds true.

Our design objective involves in specifying an updated law of $\Theta(t)$ for fuzzy logic system $\xi(x) \Theta(t)$ and a control law $u_{e}(t)$ for the tracking error dynamic in (37) such that the robust minimax tracking performance (39) or (40) is guaranteed. The updated law for $u_{e}(t)$ plays a prominent role in rough tuning and the specification of $u_{e}(t)$ plays a role in fine tuning for the robust tracking control of the uncertain robotic system in (11). A more detailed description is given in the next section.

\section{Minimax Tracking OF Robotic Systems VIA ADAPTIVE FUZZY CANCELLATION SCHEME}

From the analysis in the above section, the robust tracking control design of uncertain robotic systems is formulated as a minimax tracking control problem via an adaptive fuzzy control $u_{f}(x, \Theta)=\xi(x) \Theta(t)$ to eliminate uncertainties $f(x)$ as fully as possible and then via a minimax control $u_{e}(t)$ to attenuate the worst-case influence of $w(t)$ on tracking error below a prescribed level $\rho$. The first step in our design involves specifying an updated law for $\Theta(t)$ and a control law $u_{e}(t)$ such that for any $w(t) \in L_{2}\left[0, t_{f}\right]$ the following minimax problem is achieved, i.e.:

$$
\begin{aligned}
& \min _{u_{e}(t) \in L_{2}\left[0, t_{f}\right]} \max _{w(t) \in L_{2}\left[0, t_{f}\right]} \int_{0}^{t_{f}}\left(e^{T}(t) Q e(t)\right. \\
& +u_{e}^{T}(t) R u_{e}(t) \\
& \left.-\rho^{2} w^{T}(t) w(t)\right) d t \\
& \leq e^{T}(0) P e(0)+\frac{1}{\gamma} \tilde{\Theta}^{T}(0) \tilde{\Theta}(0)
\end{aligned}
$$

Subject to the tracking error dynamic equation in (37), let us define the cost function

$$
\begin{aligned}
J\left(e, u_{e}, w\right)= & \int_{0}^{t_{f}} e^{T}(t) Q e(t)+u_{e}^{T}(t) R u_{e}(t) \\
& -\rho^{2} w^{T}(t) w(t) d t
\end{aligned}
$$

Following some arrangement, we obtain

$$
\begin{aligned}
J\left(e, u_{e}, w\right)= & e^{T}(0) P e(0)-e^{T}\left(t_{f}\right) P e\left(t_{f}\right) \\
& +\frac{1}{\gamma} \tilde{\Theta}^{T}(0) \tilde{\Theta}(0)-\frac{1}{\gamma} \tilde{\Theta}^{T}\left(t_{f}\right) \tilde{\Theta}\left(t_{f}\right) \\
& +\int_{0}^{t_{f}}\left[e^{T}(t) Q e(t)+u_{e}^{T}(t) R u_{e}(t)\right. \\
& -\rho^{2} w^{T}(t) w(t)+\frac{d}{d t}\left(e^{T}(t) P e(t)\right) \\
& \left.+\frac{1}{\gamma} \frac{d}{d t}\left(\tilde{\Theta}^{T}(t) \tilde{\Theta}(t)\right)\right] d t \\
= & e^{T}(0) P e(0)-e^{T}\left(t_{f}\right) P e\left(t_{f}\right) \\
& +\frac{1}{\gamma} \tilde{\Theta}^{T}(0) \tilde{\Theta}(0)-\frac{1}{\gamma} \tilde{\Theta}^{T}\left(t_{f}\right) \tilde{\Theta}\left(t_{f}\right) \\
& +\int_{0}^{t_{f}}\left[e^{T}(t) Q e(t)+u_{e}^{T}(t) R u_{e}(t)\right. \\
& -\rho^{2} w^{T}(t) w(t)+\dot{e}^{T}(t) P e(t) \\
& +e^{T}(t) P \dot{e}(t)+\frac{1}{\gamma} \dot{\tilde{\Theta}}^{T}(t) \tilde{\Theta}(t) \\
& \left.+\frac{1}{\gamma} \tilde{\Theta}^{T}(t) \dot{\tilde{\Theta}}(t)\right] d t \\
&
\end{aligned}
$$

Substituting (37) into the above equation yields

$$
\begin{aligned}
J\left(e, u_{e}, w\right)= & e^{T}(0) P e(0)-e^{T}\left(t_{f}\right) P e\left(t_{f}\right) \\
& +\frac{1}{\gamma} \tilde{\Theta}^{T}(0) \tilde{\Theta}(0)-\frac{1}{\gamma} \tilde{\Theta}^{T}\left(t_{f}\right) \tilde{\Theta}\left(t_{f}\right) \\
& +\int_{0}^{t_{f}}\left[e^{T}(t)\left(A^{T} P+P A+Q\right) e(t)\right. \\
& +u_{e}^{T}(t) R u_{e}(t)-\rho^{2} w^{T}(t) w(t) \\
& +u_{e}^{T}(t) B^{T} P e(t)+e^{T}(t) P B u_{e}(t) \\
& +e^{T}(t) P B \xi(x) \tilde{\Theta}(t) \\
& +\tilde{\Theta}^{T}(t) \xi^{T}(x) B^{T} P e(t)+e^{T}(t) P B w(t) \\
& +w^{T}(t) B^{T} P e(t)+\frac{1}{\gamma} \dot{\tilde{\Theta}}^{T}(t) \tilde{\Theta}(t) \\
& \left.+\frac{1}{\gamma} \tilde{\Theta}^{T}(t) \dot{\tilde{\Theta}}(t)\right] d t
\end{aligned}
$$

then we get the following main result.

Theorem: For the uncertain robotic system (15) or (16), if the control $u(t)$ is chosen as

$$
\begin{aligned}
u(t)= & M_{0}\left(q_{d}^{(3)}-K_{1} \tilde{q}-K_{2} \dot{\tilde{q}}-K_{3} \ddot{\tilde{q}}+u_{e}-\xi \Theta\right) \\
& +D_{0}(q, \dot{q}, \ddot{q})
\end{aligned}
$$

with

$$
\begin{aligned}
\dot{\Theta} & =\gamma \xi^{T}(x) B^{T} P e(t) \\
u_{e} & =-R^{-1} B^{T} P e(t)
\end{aligned}
$$

where $R=R^{T}>0$ is a weighting matrix and $P=P^{T}>0$ is 
the solution of the following algebraic Riccati-like equation:

$$
P A+A^{T} P+Q-P B\left(R^{-1}-\frac{1}{\rho^{2}} I\right) B^{T} P=0
$$

then the minimax tracking in (39) or (40) is guaranteed for a prescribed $\rho$ and the corresponding worst case $w^{*}(t)$ to deteriorate the tracking performance is of the form

$$
w^{*}(t)=\frac{1}{\rho^{2}} B^{T} P e(t)
$$

Remark: $w^{*}(t)$ denotes a possible disturbance in $L_{2}\left[0, t_{f}\right]$, which leads to the worst-case influence on tracking error from the $L_{2}$-norm perspective.

Proof: From (44) and after some rearrangement, we get

$$
\begin{aligned}
J\left(e, u_{e}, w\right)= & e^{T}(0) P e(0)-e^{T}\left(t_{f}\right) P e\left(t_{f}\right) \\
& +\frac{1}{\gamma} \tilde{\Theta}^{T}(0) \tilde{\Theta}(0)-\frac{1}{\gamma} \tilde{\Theta}^{T}\left(t_{f}\right) \tilde{\Theta}\left(t_{f}\right) \\
& +\int_{0}^{t_{f}}\left[e^{T}(t)\left(A^{T} P+P A+Q\right) e(t)\right. \\
& +u_{e}^{T}(t) R u_{e}(t)+u_{e}^{T}(t) B^{T} P e(t) \\
& +e^{T}(t) P B u_{e}(t)+\xi(x) e^{T}(t) P B \tilde{\Theta}(t) \\
& +\frac{1}{\gamma} \dot{\tilde{\Theta}}^{T}(t) \tilde{\Theta}(t)+\frac{1}{\gamma} \tilde{\Theta}^{T}(t) \dot{\tilde{\Theta}}(t) \\
& +\tilde{\Theta}(t) B^{T} P e(t) \xi^{T}(x)+w^{T}(t) B^{T} P e(t) \\
& \left.+e^{T}(t) P B w(t)-\rho^{2} w^{T}(t) w(t)\right] d t .
\end{aligned}
$$

From (46)-(49) we get

$$
\begin{aligned}
J\left(e, u_{e}, w\right)= & e^{T}(0) P e(0)+\frac{1}{\gamma} \tilde{\Theta}^{T}(0) \tilde{\Theta}(0) \\
& -e^{T}\left(t_{f}\right) P e\left(t_{f}\right)-\frac{1}{\gamma} \tilde{\Theta}^{T}\left(t_{f}\right) \tilde{\Theta}\left(t_{f}\right) \\
& +\int_{0}^{t_{f}}\left[e^{T}(t)\left(P A+A^{T} P+Q\right) e(t)\right. \\
& \left.+u_{e}^{T}(t) R u_{e}(t)-\rho^{2} w^{T}(t) w(t)\right] d t \\
= & e^{T}(0) P e(0)+\frac{1}{\gamma} \tilde{\Theta}^{T}(0) \Theta(0) \\
& -\frac{1}{\gamma} \tilde{\Theta}^{T}\left(t_{f}\right) \Theta\left(t_{f}\right) \\
& +\int_{0}^{t_{f}}\left[e ^ { T } ( t ) \left(P A+A^{T} P+Q\right.\right. \\
& \left.-P B\left(R-1-\frac{1}{\rho^{2}} I\right) B^{T} P\right) e(t) \\
& +u_{e}^{T}(t) R u_{e}(t)+u_{e}^{T}(t) B^{T} P e(t) \\
& +e^{T}(t) P B u_{e}(t)+e^{T}(t) P B R^{-1} P e(t) \\
& -\rho^{2} w^{T}(t) w(t)+w^{T}(t) B^{T} P e(t) \\
& +e^{T}(t) P B w(t) \\
& \left.-\frac{1}{\rho^{2}} e^{T}(t) P B B^{T} P e(t)\right] d t \\
&
\end{aligned}
$$

From (48) and using the technique of completion of the squares we get

$$
\begin{aligned}
J\left(e, u_{e}, w\right)= & e^{T}(0) \operatorname{Pe}(0)+\frac{1}{\gamma} \tilde{\Theta}^{T}(0) \tilde{\Theta}(0) \\
& -e^{T}\left(t_{f}\right) \operatorname{Pe}\left(t_{f}\right)-\frac{1}{\gamma} \tilde{\Theta}^{T}\left(t_{f}\right) \tilde{\Theta}\left(t_{f}\right) \\
& +\int_{0}^{t_{f}}\left[\left(R u_{e}(t)+B^{T} \operatorname{Pe}(t)\right)^{T} R^{-1}\right. \\
& \times\left(R u_{e}(t)+B^{T} \operatorname{Pe}(t)\right) \\
& -\left(\rho w(t)-\frac{1}{\rho} B^{T} \operatorname{Pe}(t)\right)^{T} \\
& \left.\times\left(\rho w(t)-\frac{1}{\rho} B^{T} \operatorname{Pe}(t)\right)\right] d t
\end{aligned}
$$

From (52) and the dynamic game theory [13], [14], [27] we obtain the optimal control $u_{e}(t)$ as (47) and the worst case $w^{*}(t)$ as (49). Then

$$
\begin{aligned}
\min _{u_{e}(t)} \max _{w(t)} J\left(e, u_{e}, w\right) & \\
= & e^{T}(0) \operatorname{Pe}(0)+\frac{1}{\gamma} \tilde{\Theta}^{T}(0) \tilde{\Theta}(0) \\
& -e^{T}\left(t_{f}\right) \operatorname{Pe}\left(t_{f}\right)-\frac{1}{\gamma} \tilde{\Theta}^{T}\left(t_{f}\right) \tilde{\Theta}\left(t_{f}\right) \\
& \leq e^{T}(0) \operatorname{Pe}(0)+\frac{1}{\gamma} \tilde{\Theta}^{T}(0) \tilde{\Theta}(0) .
\end{aligned}
$$

The above inequality holds by the fact that $P=$ $P^{T}>0, R=R^{T}>0$ and $\gamma>0$. From (42) it is seen

$$
\begin{aligned}
\min _{u_{e}(t)} & \max _{w(t)} \int_{0}^{t_{f}}\left(e^{T}(t) Q e(t)+u_{e}^{T}(t) R u_{e}(t)\right. \\
& \left.-\rho^{2} w^{T}(t) w(t)\right) d t \\
\leq & e^{T}(0) \operatorname{Pe}(0)+\frac{1}{\gamma} \tilde{\Theta}^{T}(0) \tilde{\Theta}(0)
\end{aligned}
$$

This is (41): if $e(0)=0$ and $\tilde{\Theta}(0)=0$, then (54) is reduced to (40).

\section{Remarks:}

1) To guarantee the positive definite solution of $P$ in the algebraic Riccati-like equation (48), the following constraint must hold [13]:

$$
R^{-1}-\frac{1}{\rho^{2}} I \geq 0 \quad \text { or } \rho^{2} I \geq R
$$

For a prescribed attenuation level $\rho$, the weighting matrix $R$ on the control $u_{e}(t)$ in (47) must satisfy the above constraint to guarantee the solvability of minimax tracking control of the uncertain robotic system in (11). Hence, a robust tracking design with an arbitrary attenuation of $w(t)$ is possible by the proposed method with an adequate choice of $R$. However, if the attenuation level $\rho$ is specified as an extremely small value, from (55) $R^{-1}$ must be of an extremely large value. Under this circumstance, $u_{e}(t)$ may require a large control energy. There is a tradeoff between $\rho$ and $u_{e}(t)$.

2) Notably, $\Omega$ and $\Omega_{e}$ do not need to be known or specified beforehand. Since $w(t)$ is bounded by the universal approximation theorem [25] and the assumption of 
bounded external disturbances, $e(t)$ is bounded. Moreover, additional tools concerning projection algorithm [21] can be used to analyze the bounded problems of $\Theta(t)$. Assume that the constraint set $\Omega$ is specified as $\Omega \triangleq\{\Theta \mid\|\Theta\| \leq M\}$, where $M$ is a positive constant. Then, the parameter update law in (46) must be modified as [21]

$$
\dot{\Theta}=\gamma \dot{\xi}^{T}(x) B^{T} P e(t)-U(\Theta)
$$

where

$$
U(\Theta)=\left\{\begin{array}{c}
0 \\
\text { if }\|\Theta\|<M \quad \text { or }(\|\Theta\|<M \\
\text { and } \left.\Theta^{T} \xi^{T} B^{T} \operatorname{Pe}(t) \leq 0\right) \\
\gamma \Theta \frac{\Theta^{T} \xi^{T}(x) B^{T} \operatorname{Pe}(t)}{\|\Theta\|^{2}} \\
\text { if } \left.\|\Theta\| \geq M \text { and } \Theta^{T} \xi^{T} B^{T} \operatorname{Pe}(t)>0\right) .
\end{array}\right.
$$

Since $\left(e^{T}(t) P B \xi(x) \tilde{\Theta}(t)\right)+(1 / \gamma)\left(\dot{\tilde{\Theta}}^{T}(t) \tilde{\Theta}(t)\right)=$ $\left(e^{T}(t) P B \xi^{T}(x) \Theta(t) /\|\Theta(t)\|^{2}\right) \Theta^{T}(t) \tilde{\Theta}(t)$, it can be shown by geometric argument that the angle between $\widetilde{\Theta}(t)$ and $\Theta(t)$ is larger than $\pi / 2$ at projection case. So $\tilde{\Theta}^{T}(t) \Theta(t)<0$. We obtain $e^{T}(t) P B \xi(x) \tilde{\Theta}(t)+\frac{1}{\gamma} \dot{\tilde{\Theta}}^{T}(t) \tilde{\Theta}(t) \leq 0$ and $\tilde{\Theta}^{T}(t) \xi^{T}(x) B^{T} P e(t)+(1 / \gamma) \tilde{\Theta}^{T}(t) \tilde{\Theta}(t) \leq 0$. By the same procedure as the proof in the Theorem, the minimax tracking performance (41) can also be guaranteed via the updated law (56).

From above analysis, a design procedure for the minimax tracking of uncertain robotic systems with motor driving is outlined as follows.

Design Procedure:

Step 1 Specify $K_{1}, K_{2}$, and $K_{3}$ to determine matrix $A$ with desired eigenvalues.

Step 2 Determine the fuzzy architecture $\xi(x)$ and specify the desired attenuation level $\rho$.

Step 3 Select positive-definite weighting matrices $Q$ and $R$ with $R \leq \rho^{2} I$ to guarantee the solvability of the minimax tracking.

Step 4 Solve the positive-definite matrix $P$ from the Riccati-like equation (48).

Step 5 Compute the minimax tracking control law (47) and the parameter update law in (46) and then $u(t)$ in (45).

In the next section, a design example is given according to the above design procedure to demonstrate the effectiveness of the proposed design method.

\section{A Simulation Example}

In this section, we test our proposed fuzzy-based minimax control on the robust tracking design of a two-link robot by using a computer. Consider a two-link manipulator driven by dc motor as Fig. 2 with system parameters as: link mass $m_{1}, m_{2}(\mathrm{~kg})$, lengths $l_{1}, l_{2}(\mathrm{~m})$, angular positions $q_{1}, q_{2}(\mathrm{rad})$.

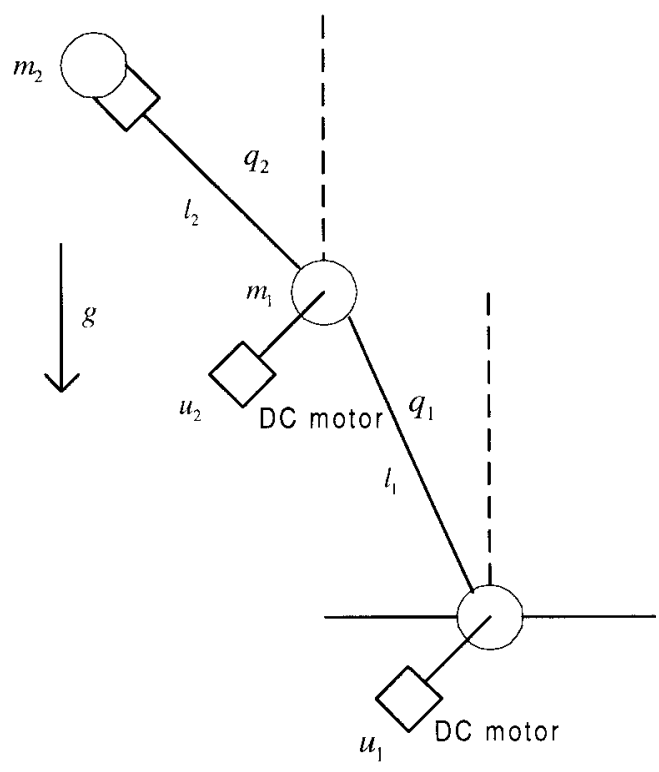

Fig. 2. The two-link robotic manipulator driven by dc motors.

The parameters for the equation of motion (1) are

$$
\begin{aligned}
& M^{\prime}(q)=\left[\begin{array}{cc}
\left(m_{1}+m_{2}\right) l_{1}^{2} & m_{2} l_{1} l_{2}\left(s_{1} s_{2}+c_{1} c_{2}\right) \\
m_{2} l_{1} l_{2}\left(s_{1} s_{2}+c_{1} c_{2}\right) & m_{2} l_{2}^{2}
\end{array}\right] \\
& C(q, \dot{q})=m_{2} l_{1} l_{2}\left(c_{1} s_{2}-s_{1} c_{2}\right)\left[\begin{array}{cc}
0 & -\dot{q}_{2} \\
-\dot{q}_{1} & 0
\end{array}\right] \\
& G(q)=\left[\begin{array}{c}
-\left(m_{1}+m_{2}\right) l_{1} g s_{1} \\
-m_{2} l_{2} g s_{2}
\end{array}\right]
\end{aligned}
$$

where $q \in R^{2}$ and the shorthand notations $c_{1}=\cos \left(q_{1}\right), c_{2}=$ $\cos \left(q_{2}\right), s_{1}=\sin \left(q_{1}\right)$ and $s_{2}=\sin \left(q_{2}\right)$ are used.

Assume that the trajectory planning problem for a weightlifting operation is considered and the two-link manipulator suffers from time-varying parametric uncertainties and exogenous disturbances.

- The resistance matrix of the armature circuit is

$$
R_{t}=\left[\begin{array}{cc}
1.6 & 0 \\
0 & 1.6
\end{array}\right]
$$

- The inductance matrix of the armature circuit is

$$
L=\left[\begin{array}{cc}
0.048 & 0 \\
0 & 0.048
\end{array}\right]
$$

- The back EMF constant of the motor is

$$
K_{b}=\left[\begin{array}{cc}
0.19 & 0 \\
0 & 0.19
\end{array}\right] \text {. }
$$

- The motor torque constant is

$$
K_{t}=\left[\begin{array}{cc}
0.2613 & 0 \\
0 & 0.2613
\end{array}\right]
$$

- The gear ratio is

$$
N=\left[\begin{array}{cc}
62.55 & 0 \\
0 & 107.81
\end{array}\right] .
$$


The proposed adaptive fuzzy-based model reference control with the minimax tracking performance is employed to treat this robust robotic trajectory planning problem. For the convenience of simulation, the nominal parameters of the robotic system are given as $m_{1}=4.6(\mathrm{~kg}), m_{2}=2.3(\mathrm{~kg}), l_{1}=0.5(\mathrm{~m})$, $l_{2}=0.2(\mathrm{~m}), g=9.8 \mathrm{~m} / \mathrm{s}^{2}$, and the initial conditions $q_{1}(0)=$ $2, q_{2}(0)=1, \dot{q}_{1}(0)=\dot{q}_{2}(0)=\ddot{q}_{1}(0)=\ddot{q}_{2}(0)=0$. The desired reference trajectories are $q_{1 d}(t)=\sin (t), q_{2 d}(t)=\cos (t)$, respectively.

Assume that the parameters $m_{1}$ and $m_{2}$ be perturbed in the following form:

$$
\Delta m_{1}(t)=0.1 \sin (2 t) \text { and } \Delta m_{2}(t)=0.1 \sin (2 t)
$$

respectively. Moreover, the exogenous disturbances $d_{1}$ and $d_{2}$ are assumed as

$$
d_{1}=0.1 \sin (2 t), \quad d_{2}=0.05 \sin (2 t) .
$$

Obviously, the parameter uncertainties and exogenous disturbances are extremely large. Therefore, the proposed fuzzybased minimax tracking control algorithm is employed to treat this robust tracking control design.

Now, following the design procedure in the above section, the robust tracking control design is given by the following steps.

Step 1 Specify

$$
K_{1}=\left[\begin{array}{ll}
9 & 0 \\
0 & 7
\end{array}\right], K_{2}=\left[\begin{array}{cc}
10 & 0 \\
0 & 14
\end{array}\right], K_{3}=\left[\begin{array}{ll}
5 & 0 \\
0 & 8
\end{array}\right]
$$

such that the eigenvalues of the nominal tracking system are $-1.3037+1.4359 i,-1.3037-1.4359 i$, $-2.3926,-5.7913,-1.2087$, and -1.0000 .

Step 2 The following membership functions are selected:

$$
\begin{aligned}
& \mu_{F_{i}^{1}}=\exp \left[-\left(\frac{\left(x_{i}-3 \times a_{i}\right)}{b_{i}}\right)^{2}\right] \\
& \mu_{F_{i}^{2}}=\exp \left[-\left(\frac{\left(x_{i}-2 \times a_{i}\right)}{b_{i}}\right)^{2}\right] \\
& \mu_{F_{i}^{3}}=\exp \left[-\left(\frac{\left(x_{i}-1 \times a_{i}\right)}{b_{i}}\right)^{2}\right] \\
& \mu_{F_{i}^{4}}=\exp \left[-\left(\frac{x_{i}}{b_{i}}\right)^{2}\right] \\
& \mu_{F_{i}^{5}}=\exp \left[-\left(\frac{\left(x_{i}+1 \times a_{i}\right)}{b_{i}}\right)^{2}\right] \\
& \mu_{F_{i}^{6}}=\exp \left[-\left(\frac{\left(x_{i}+2 \times a_{i}\right)}{b_{i}}\right)^{2}\right] \\
& \mu_{F_{i}^{7}}=\exp \left[-\left(\frac{\left(x_{i}+3 \times a_{i}\right)}{b_{i}}\right)^{2}\right]
\end{aligned}
$$

where $i=1,2,3$, and $a_{1}=0.5, a_{2}=1, a_{3}=2$, $b_{1}=10, b_{2}=20, b_{3}=30$. Because the system has six state variables, forty-two fuzzy rules of the following form are included in the fuzzy rule bases. Denote

$R^{l}: \quad$ If $x_{1}$ is $F_{1}^{j}$, then $u_{f}$ is $G_{1}^{j}$ for $j=1,2, \cdots 7$ and $l=1,2, \cdots 7$.

$R^{l}: \quad$ If $x_{2}$ is $F_{2}^{j}$, then $u_{f}$ is $G_{2}^{j}$ for $j=1,2, \cdots 7$ and $l=8,9, \cdots 14$.

$R^{l}: \quad$ If $x_{3}$ is $F_{3}^{j}$, then $u_{f}$ is $G_{3}^{j}$ for $j=1,2, \cdots 7$ and $l=15,16, \cdots 21$.

$R^{l}: \quad$ If $x_{4}$ is $F_{4}^{j}$, then $u_{f}$ is $G_{4}^{j}$ for $j=1,2, \cdots 7$ and $l=22,23, \cdots 28$.

$R^{l}: \quad$ If $x_{5}$ is $F_{5}^{j}$, then $u_{f}$ is $G_{5}^{j}$ for $j=1,2, \cdots 7$ and $l=29,30, \cdots 35$.

$R^{l}: \quad$ If $x_{6}$ is $F_{6}^{j}$, then $u_{f}$ is $G_{6}^{j}$ for $j=1,2, \cdots 7$ and $l=36,37, \cdots 42$.

Denote

$D_{m}=\sum_{j=1}^{7} \prod_{i=1}^{3} \mu_{F_{i}^{j}}\left(x_{i}\right)$

$\varsigma(x)=\left[\begin{array}{ccc}\prod_{i=1}^{3} \mu_{F_{i}^{1}}\left(x_{i}\right) & & \prod_{i=1}^{3} \mu_{F_{i}^{7}}\left(x_{i}\right) \\ D_{m} & \ldots & \\ D_{m}\end{array}\right]$

$\xi(x)=\left[\begin{array}{cc}\varsigma(x) & 0 \\ 0 & \varsigma(x)\end{array}\right]$

$\Theta(t)=\left[\begin{array}{llllllll}\theta_{11} & \theta_{12} & \cdots & \theta_{17} & \theta_{21} & \theta_{22} & \cdots & \theta_{27}\end{array}\right]$.

The attenuation level $\rho$ is selected as $\rho=\infty, \rho=$ 0.1 , and $\rho=0.05$, respectively. In the case of $\rho=\infty$ (i.e., the $H_{2}$ optimal tracking control is used but without attenuation of $w(t)$ ), we only want to reveal the deterioration of tracking performance by $w(t)$.

Step 3 Select weighting matrix $Q=\operatorname{diag}\left[100 I_{2}, 10 I_{2}, I_{2}\right]$, and $R=\rho^{2} I$ to guarantee the solvability of the minimax tracking problem [see (55)].

Step 4 Solve the Riccati-like equation (48). In the $\rho=\infty$ case, we can solve

$$
P=\left[\begin{array}{cccccc}
17.35 & 0 & 10.05 & 0 & 0.031 & 0 \\
0 & 17.35 & 0 & 10.05 & 0 & 0.031 \\
10.05 & 0 & 17.41 & 0 & 0.054 & 0 \\
0 & 10.05 & 0 & 17.42 & 0 & 0.054 \\
0.031 & 0 & 0.054 & 0 & 0.031 & 0 \\
0 & 0.031 & 0 & 0.054 & 0 & 0.031
\end{array}\right] .
$$

In the cases of $\rho=0.1$ and $\rho=0.05$, we can solve

$$
P=\left[\begin{array}{cccccc}
23.97 & 0 & 15.46 & 0 & 0.556 & 0 \\
0 & 18.05 & 0 & 11.10 & 0 & 0.714 \\
15.46 & 0 & 11.10 & 0 & 2.046 & 0 \\
0 & 11.10 & 0 & 19.24 & 0 & 1.150 \\
0.556 & 0 & 2.046 & 0 & 1.409 & 0 \\
0 & 0.714 & 0 & 1.409 & 0 & 0.769
\end{array}\right] .
$$



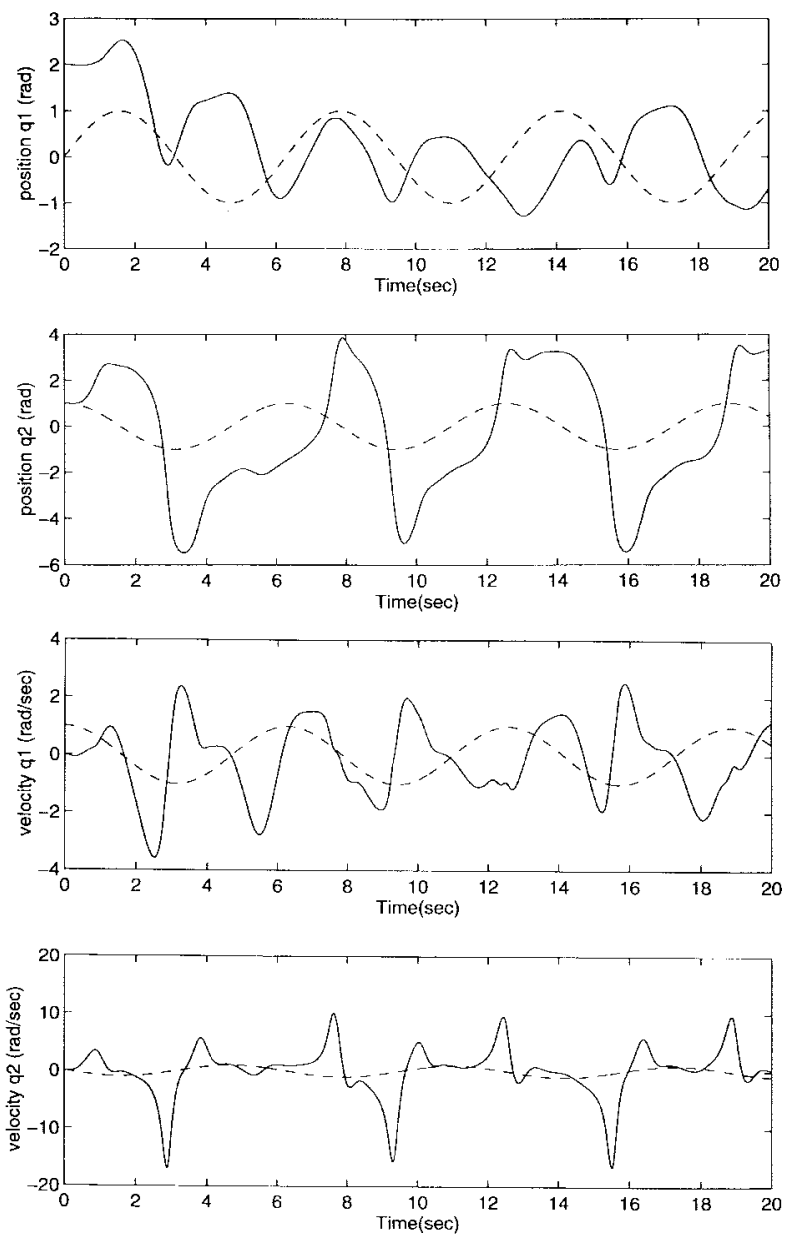

Fig. 3. The $\mathrm{H}_{2}$ optimal tracking results without attenuation of $w(t)$ (desired trajectory: “," actual trajectory: “__”).

In this simulation, we use the function "are" in the WINDOW MATLAB to solve the algebraic Riccatilike equation.

By using the control algorithms (45)-(47) to obtain the minimax tracking controller $u_{e}(t)$, the voltage control input $u(t)$ and the update law for $\Theta(t)$ are obtained as follows:

Step 5 In the $\rho=\infty$ case, the minimax tracking controller is of the following form:

$$
\begin{aligned}
\dot{\Theta}= & \xi^{T}(t)\left[\begin{array}{c}
\Theta_{1} \\
\Theta_{2}
\end{array}\right]-U(\Theta) \\
\Theta_{1}= & -0.0307\left(q_{1}-q_{1 d}\right)-0.0539\left(\dot{q}_{1}-\dot{q}_{1 d}\right) \\
& -0.0313\left(\ddot{q}_{1}-\ddot{q}_{1 d}\right) \\
\Theta_{2}= & -0.0309\left(q_{2}-q_{2 d}\right)-0.0539\left(\dot{q}_{2}-\dot{q}_{2 d}\right) \\
& -0.0310\left(\ddot{q}_{2}-\ddot{q}_{2 d}\right) \\
u_{f}(t)= & {\left[\begin{array}{l}
u_{f_{1}}(t) \\
u_{f_{2}}(t)
\end{array}\right]=\xi(x) \Theta(t) }
\end{aligned}
$$

and

$$
u(t)=\left[\begin{array}{ll}
0.0051 & 0.0004 \\
0.0002 & 0.0002
\end{array}\right]\left[\begin{array}{l}
u_{1}(t) \\
u_{2}(t)
\end{array}\right]+D_{0}
$$
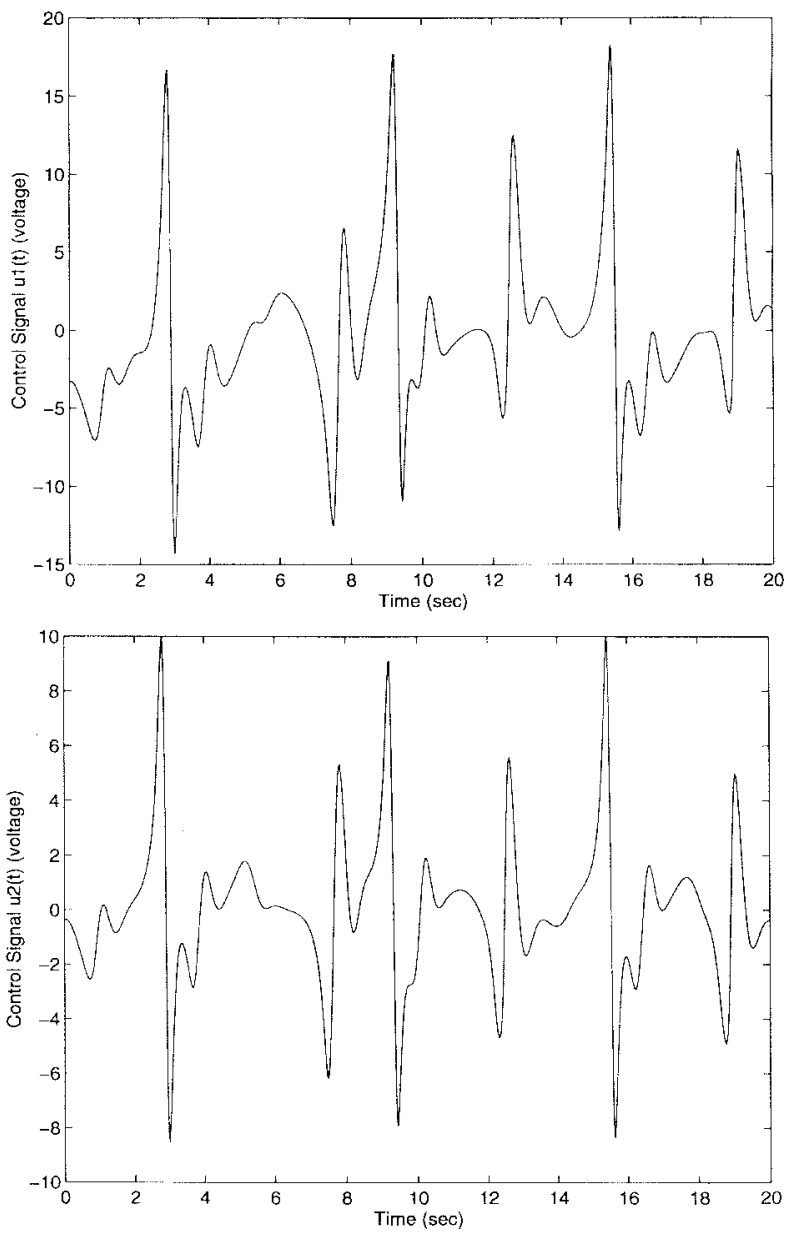

Fig. 4. Control signals of the $\mathrm{H}_{2}$ optimal tracking case.

$$
\begin{aligned}
u_{1}(t)= & q_{1 d}^{(3)}-9\left(q_{1}-q_{1 d}\right)-10\left(\dot{q}_{1}-\dot{q}_{1 d}\right) \\
& -5\left(\ddot{q}_{1}-\ddot{q}_{1 d}\right)-u_{f_{1}} \\
u_{2}(t)= & q_{2 d}^{(3)}-7\left(q_{2}-q_{2 d}\right)-14\left(\dot{q}_{2}-\dot{q}_{2 d}\right) \\
& -8\left(\ddot{q}_{2}-\ddot{q}_{2 d}\right)-u_{f_{2}}
\end{aligned}
$$

where $D_{0}$ is the nominal value of $D(q, \dot{q}, \ddot{q})$ in (13).

In the $\rho=0.1$ case, the minimax tracking controller is obtained as

$$
\begin{aligned}
\dot{\Theta}= & \xi^{T}(t)\left[\begin{array}{l}
\Theta_{1} \\
\Theta_{2}
\end{array}\right]-U(\Theta) \\
\Theta_{1}= & -0.5556\left(q_{1}-q_{1 d}\right)-2.0461\left(\dot{q}_{1}-\dot{q}_{1 d}\right) \\
& -1.4092\left(\ddot{q}_{1}-\ddot{q}_{1 d}\right) \\
\Theta_{2}= & -0.7143\left(q_{2}-q_{2 d}\right)-1.1497\left(\dot{q}_{2}-\dot{q}_{2 d}\right) \\
& -0.7687\left(\ddot{q}_{2}-\ddot{q}_{2 d}\right) \\
u_{e_{1}}= & -55.5556\left(q_{1}-q_{1 d}\right)-204.6070\left(\dot{q}_{1}-\dot{q}_{1 d}\right) \\
& -140.9214\left(\ddot{q}_{1}-\ddot{q}_{1 d}\right) \\
u_{e_{2}}= & -71.4286\left(q_{2}-q_{2 d}\right)-114.9660\left(\dot{q}_{2}-\dot{q}_{2 d}\right) \\
& -76.8707\left(\ddot{q}_{2}-\ddot{q}_{2 d}\right) \\
u_{f}(t)= & {\left[\begin{array}{l}
u_{f_{1}}(t) \\
u_{f_{2}}(t)
\end{array}\right]=\xi(x) \Theta(t) }
\end{aligned}
$$



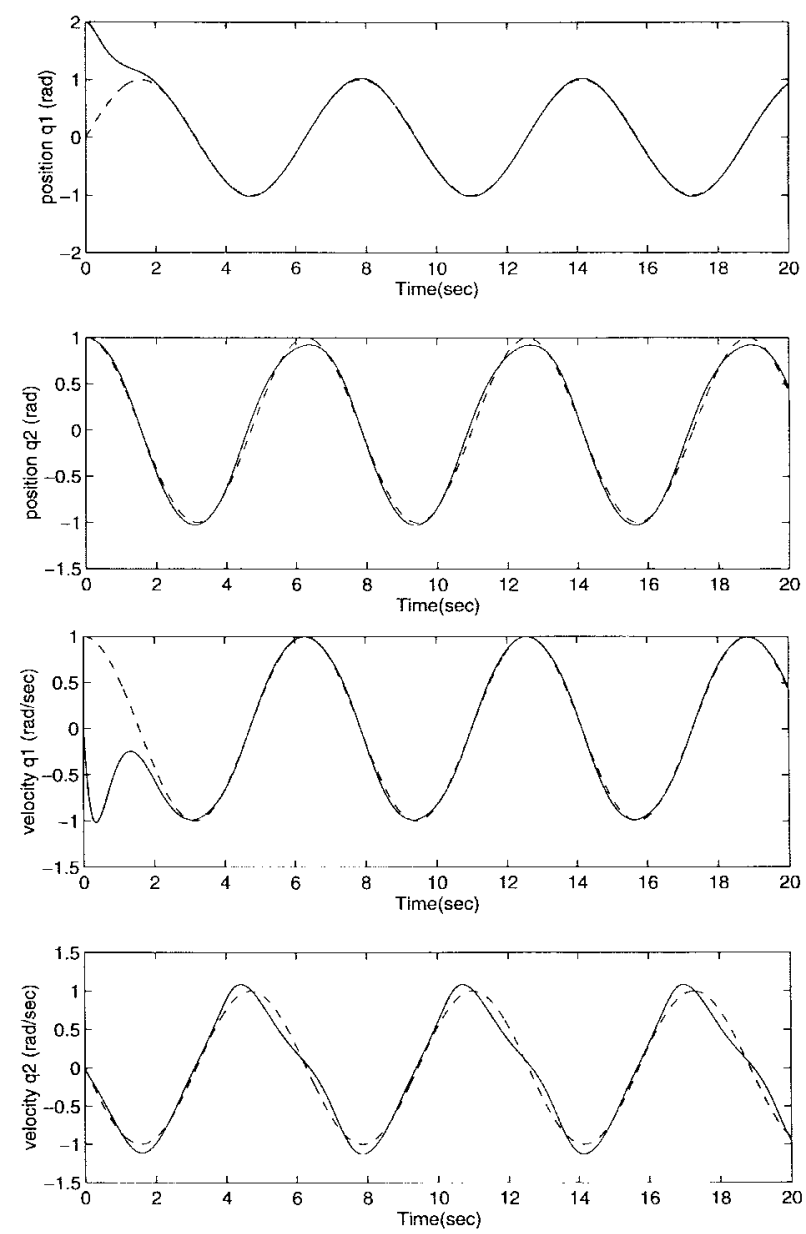

Fig. 5. Robust $H_{\infty}$ tracking results with attenuation level $\rho=0.1$ (desired trajectory: "," actual trajectory: “__”).

and

$$
\begin{aligned}
u(t)= & {\left[\begin{array}{ll}
0.0051 & 0.0004 \\
0.0002 & 0.0002
\end{array}\right]\left[\begin{array}{l}
u_{1}(t) \\
u_{2}(t)
\end{array}\right]+D_{0} } \\
u_{1}(t)= & q_{1 d}^{(3)}-9\left(q_{1}-q_{1 d}\right)-10\left(\dot{q}_{1}-\dot{q}_{1 d}\right) \\
& -5\left(\ddot{q}_{1}-\ddot{q}_{1 d}\right)-u_{f_{1}} \\
u_{2}(t)= & q_{2 d}^{(3)}-7\left(q_{2}-q_{2 d}\right)-14\left(\dot{q}_{2}-\dot{q}_{2 d}\right) \\
& -8\left(\ddot{q}_{2}-\ddot{q}_{2 d}\right)-u_{f_{2}} .
\end{aligned}
$$

In the $\rho=0.05$ case, the minimax tracking controller is of the following form:

$$
\begin{aligned}
\dot{\Theta}= & \xi^{T}(t)\left[\begin{array}{l}
\Theta_{1} \\
\Theta_{2}
\end{array}\right]-U(\Theta) \\
\Theta_{1}= & -0.5556\left(q_{1}-q_{1 d}\right)-2.0461\left(\dot{q}_{1}-\dot{q}_{1 d}\right) \\
& -1.4092\left(\ddot{q}_{1}-\ddot{q}_{1 d}\right) \\
\Theta_{2}= & -0.7143\left(q_{2}-q_{2 d}\right)-1.1497\left(\dot{q}_{2}-\dot{q}_{2 d}\right) \\
& -0.7687\left(\ddot{q}_{2}-\ddot{q}_{2 d}\right) \\
u_{e_{1}}= & -222.2222\left(q_{1}-q_{1 d}\right)-818.4282\left(\dot{q}_{1}-\dot{q}_{1 d}\right) \\
& -563.6856\left(\ddot{q}_{1}-\ddot{q}_{1 d}\right) \\
u_{e_{2}}= & -285.7143\left(q_{2}-q_{2 d}\right)-459.8639\left(\dot{q}_{2}-\dot{q}_{2 d}\right) \\
& -307.4830\left(\ddot{q}_{2}-\ddot{q}_{2 d}\right), \\
u_{f}(t)= & {\left[\begin{array}{l}
u_{f_{1}}(t) \\
u_{f_{2}}(t)
\end{array}\right]=\xi(x) \Theta(t) }
\end{aligned}
$$
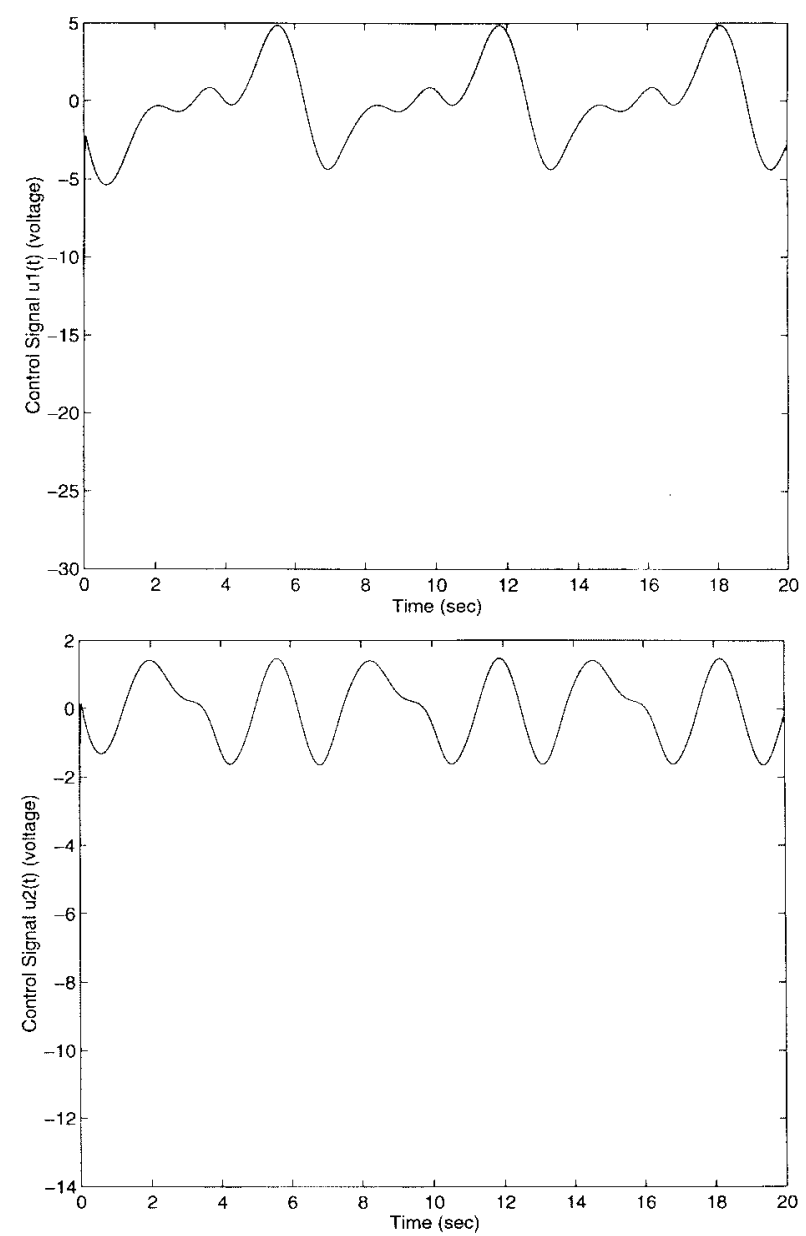

Fig. 6. Control signals with case $\rho=0.1$.

and

$$
\begin{aligned}
u(t)= & {\left[\begin{array}{ll}
0.0051 & 0.0004 \\
0.0002 & 0.0002
\end{array}\right]\left[\begin{array}{l}
u_{1}(t) \\
u_{2}(t)
\end{array}\right]+D_{0} } \\
u_{1}(t)= & q_{1 d}^{(3)}-9\left(q_{1}-q_{1 d}\right)-10\left(\dot{q}_{1}-\dot{q}_{1 d}\right) \\
& -5\left(\ddot{q}_{1}-\ddot{q}_{1 d}\right)-u_{f_{1}} \\
u_{2}(t)= & q_{2 d}^{(3)}-7\left(q_{2}-q_{2 d}\right)-14\left(\dot{q}_{2}-\dot{q}_{2 d}\right) \\
& -8\left(\ddot{q}_{2}-\ddot{q}_{2 d}\right)-u_{f_{2}} .
\end{aligned}
$$

Figs. 3-8 present the simulation results. Results obtained from the $\mathrm{H}_{2}$ optimal tracking case (i.e., $\rho=\infty$ ) are shown in Fig. 3. In Figs. 5 and 7, simulation results of the proposed adaptive fuzzy-based minimax tracking have demonstrated the tracking performance for attenuation levels $\rho=0.1$ and 0.05 , respectively. Figs. 4, 6, and 8 show the control inputs under different attenuation levels. Figs. 9 and 10 show the fluctuations of the adaptive parameters for the cases of $\rho=0.1$ and 0.05 , respectively.

According to the simulation results of the above three attenuation level cases, a specification of smaller attenuation level $\rho$ may yield a better tracking performance. In the case of $H_{2}$ optimal tracking, the effect of $w(t)$ on tracking error has not been attenuated and subsequently leads to poor tracking performance. According to these results, tracking performance 

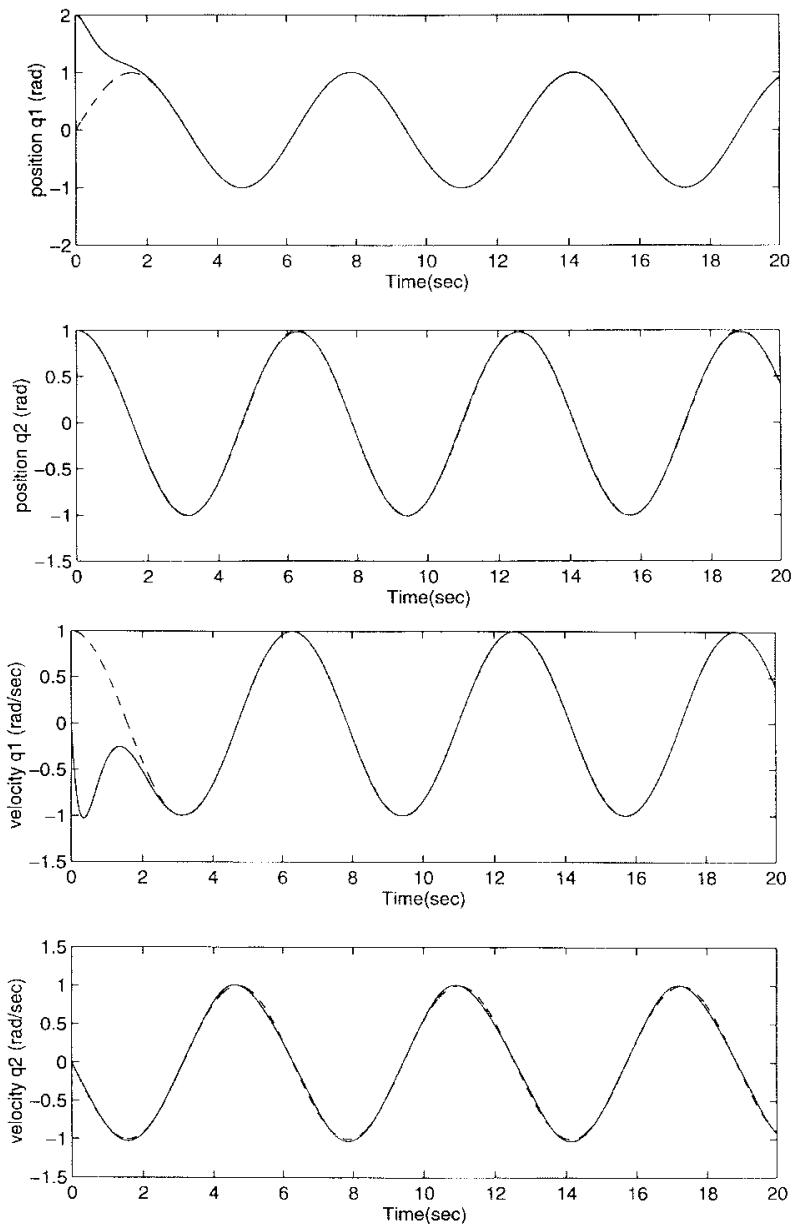

Fig. 7. Robust $H_{\infty}$ tracking results with attenuation level $\rho=0.05$ (desired trajectory: "," actual trajectory: " ”).

is unsatisfactory and even unstable. The effect of the combined disturbance $w(t)$ on tracking error is attenuated evidently as $\rho$ decreases. However, the voltage control inputs also indicate a high-gain effect of the designed controller as $\rho$ decrease, particularly, at the beginning of control. In practical control engineering design, this effect due to the residue of fuzzy cancellation and exogenous disturbance must be diminished in fuzzy-based control systems. Otherwise, it will damage the entire system, particularly, in using a small number of membership functions. However, the attenuation level $\rho$ cannot be decreased without considering the increase of the control input. This is a tradeoff between the amplitude of control signal and the tracking performance.

In [5], the following control is employed for robust tracking of robot system with dc motor

$$
\begin{gathered}
u_{0}[e(t)]=-\rho B^{T} P_{n} e(t)-\mu(e(t)) \frac{B^{T} P_{n} e(t)}{\left\|B^{T} P_{n} e(t)\right\|+\varepsilon} \\
\varepsilon>0
\end{gathered}
$$

where $\mu(e(t))=\alpha_{1}\|e(t)\|+\alpha_{2}\|e(t)\|^{2}+\alpha_{3}\|e(t)\|^{3}+\alpha_{4}$ and $P_{n}$ is solved by the following Riccati-like equation:

$$
P_{n}\left(A+\beta_{n} I\right)+\left(A+\beta_{n} I\right)^{T} P_{n}+Q-\rho P_{n} B B^{T} P_{n}=0 .
$$
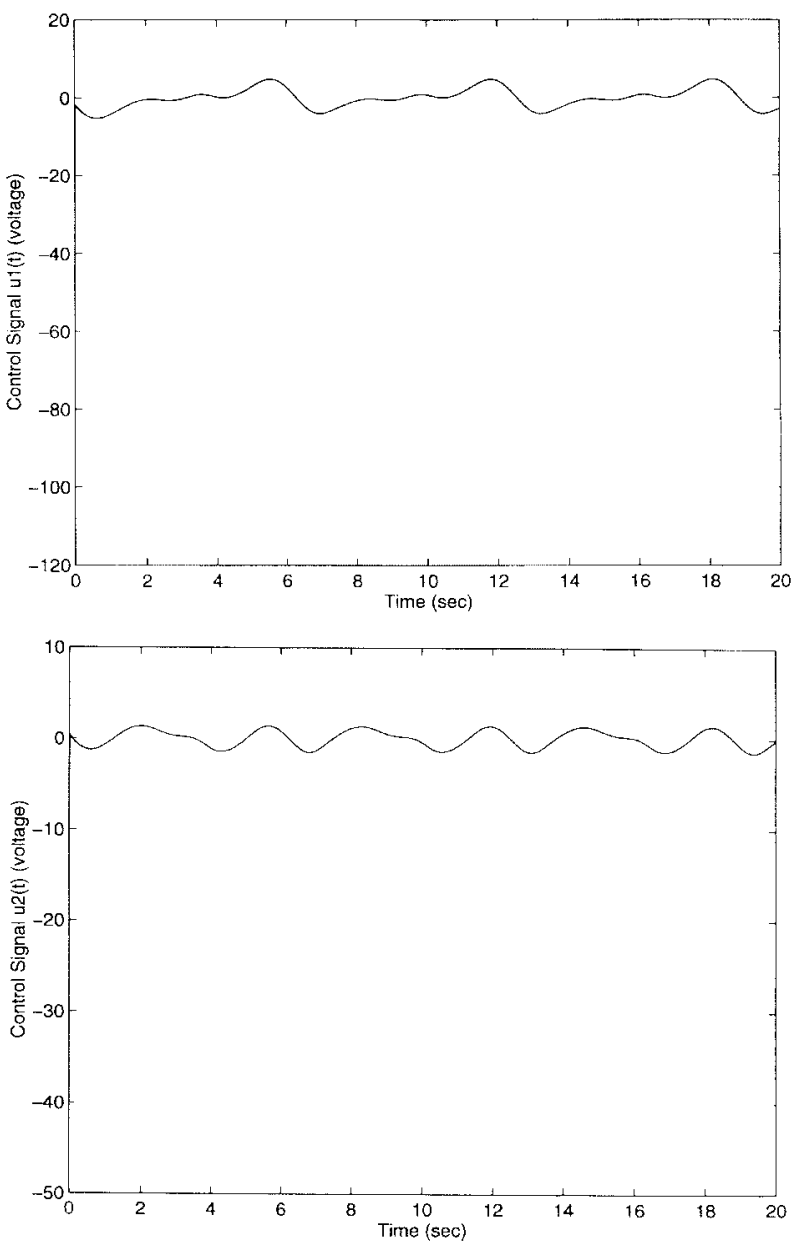

Fig. 8. Control signals with case $\rho=0.05$.

Some comparisons are given as follows:

1) In control algorithm of [5], the second term in (57) is employed to override the uncertainties or disturbance directly through a high-gain control that is proportional to $\|e(t)\|,\|e(t)\|^{2}$, and $\|e(t)\|^{3}$. If the initial $e(0)$ is not very small, it will lead to a very high-control signal and the control system will diverge. By applying the control algorithm in [5] to our simulation example, we have found the tracking system diverges even with small initial $e(0)$. Obviously, it is not a good design to override the uncertainties directly. In our method, an adaptive fuzzy scheme is employed to optimally cancel the uncertainties beforehand. Therefore, we only need $u_{e}(t)$ to eliminate the effect of residue with a little effort.

2) The choice of control parameters $\alpha_{1}, \alpha_{2}, \alpha_{3}$, and $\alpha_{4}$ in [5] are complex and case by case. However, in our case, a simple design procedure is proposed by a systematic method. Furthermore, a comparison with conventional optimal $\mathrm{H}_{2}$ tracking control without uncertain cancellation is shown in Figs. 3 and 4. From the results in Figs. 5-8, it is seen that the proposed method has much better performance than that of the conventional $\mathrm{H}_{2}$ optimal tracking control. The reason is that the effort of uncertain parameters 


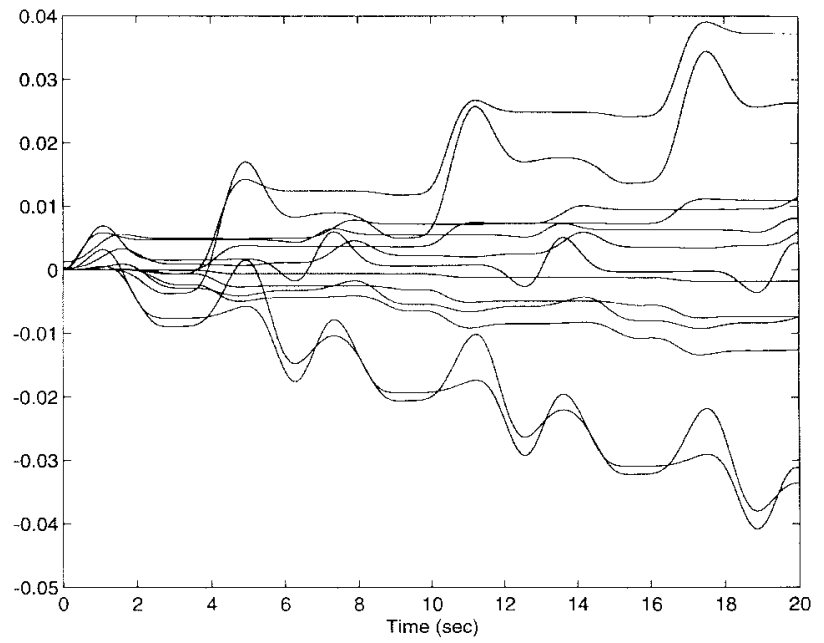

Fig. 9. The fluctuations of the adaptive parameters $\theta_{i}$ for the case of $\rho=0.1$.

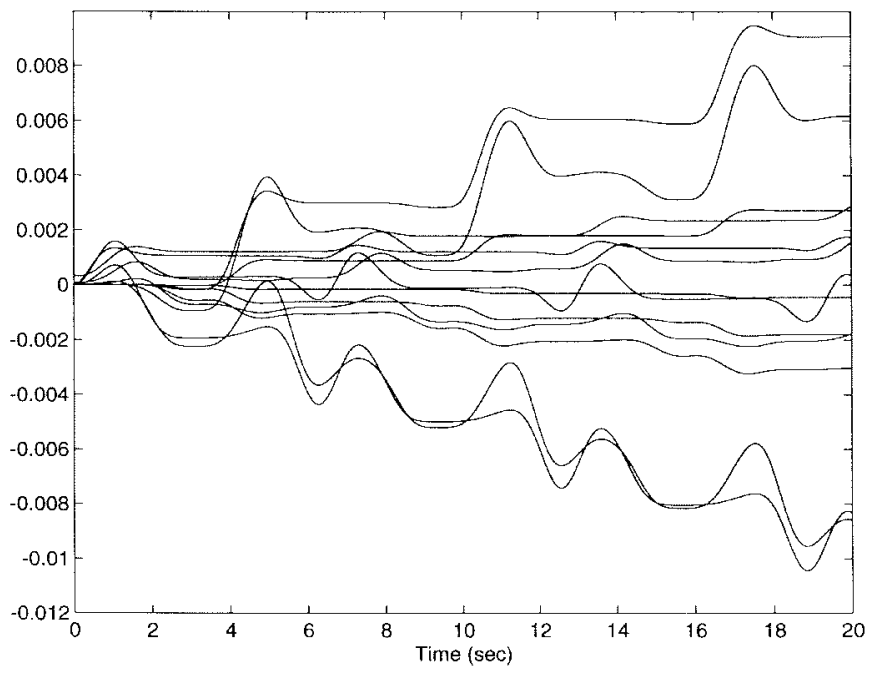

Fig. 10. The fluctuations of the adaptive parameters $\theta_{i}$ for the case of $\rho=0.05$.

and external disturbance is efficiently eliminated by the proposed fuzzy-based minimax cancellation scheme.

\section{CONCLUSION}

In this paper, an adaptive fuzzy cancellation technique and a minimax (or $H_{\infty}$ ) attenuation technique have functioned in the roles of a rough tuning and a fine tuning, respectively, and are combined together to enhance the robust tracking performance of uncertain robotic systems including motor dynamics. The solvability of this robust minimax tracking problem for uncertain robot system is also observed. According to our results, a desired minimax tracking performance can be achieved if the weighting matrix on control signal is adequately specified.

Actually, the proposed fuzzy-based minimax tracking method can be applied to any robust control design of an uncertain nonlinear mechanical system of the form in (11) or (15). With the aid of adaptive fuzzy cancellation algorithm, the minimax tracking control design can be extended from exactly known linear systems toward nonlinear uncertain systems. Furthermore, by employing minimax attenuation technique, the tracking performance of fuzzybased control design for uncertain nonlinear mechanical systems can be significantly improved. Therefore, the proposed design algorithm is appropriate for practical control design of mechanical systems with large parameter perturbations and external disturbances. The proposed design method is simple, the number of membership functions for the proposed control law can be extremely small. However, because of the use of fuzzy cancellation technique and minimax tracking scheme, the results are less conservative than the other robust control methods. Simulation results have indicated that the desired robust tracking performance of uncertain dc motordriven robotic systems can be achieved via the proposed method.

\section{REFERENCES}

[1] M. W. Spong, Robot Dynamics and Control. New York: Wiley, 1989.

[2] J. J. E. Slotine and W. Li, Applied Nonlinear Control. Englewood Cliffs, NJ: Prentice-Hall, 1991.

[3] T. J. Tarn, A. K. Bejcay, X. Yun, and Z. Li, "Effect of motor dynamics on nonlinear feedback robot arm control," IEEE Trans. Robot. Automat., vol. 7, pp. 114-122, 1991.

[4] A. J. Koivo, Fundamentals for Control of Robotic Manipulators. New York: Wiley, 1989.

[5] M. S. Mahmoud, "Robust control of robot arms including motor dynamic," Int. J. Contr., vol. 58, no. 4, pp. 853-873, 1993.

[6] R. Beekman and K. Lee, "Nonlinear robotic control including driving motor interactions," in Proc. Amer. Contr. Conf., Atlanta, GA, June 1988, pp. $1333-1338$.

[7] S. R. Pandian and M. Hanmandlu, "Adaptive generalized model-based control of robot manipulators," Int. J. Contr., vol. 58, no. 4, pp. 835-852, 1993.

[8] J. J. Craig, Adaptive Control of Mechanical Manipulators. New York: Addison-Wesley, 1988.

[9] A. Karakasoglu and S. I. Sudharsanan, "Identification and decentralized adaptive control using dynamical neural network with application to robotic manipulators," IEEE Trans. Neural Networks, vol. 4, pp. 919-930, Nov. 1993

[10] K. S. Narendra and K. Parthusarathy, "Identification and control of dynamical systems using neural networks," IEEE Trans. Neural Networks, vol. 1, pp. 4-27, June 1990

[11] B. Kosko, Neural Network and Fuzzy Systems. Englewood Cliffs, NJ: Prentice-Hall, 1992.

[12] T. Basar, $H_{\infty}$-Optimal Control and Related Minimax Design Problems. A Dynamic Game Approach. Boston, MA: Birkhäuser, 1991.

[13] A. Stoorvogel, The $H_{\infty}$ Control Problem: A State Space Approach New York: Prentice-Hall, 1992.

[14] J. Doyle, K. Glover, P. P. Khargonekar, and B. A. Francis, "State space solution to standard $H_{2}$ and $H_{\infty}$ control problems," IEEE Trans. Automatic Contr., vol. 34, pp. 831-847, Aug. 1989.

[15] B. S. Chen, T. S. Lee, and J. H. Feng, "A nonlinear $H_{\infty}$ contro design in robotic systems under parameter perturbation and external disturbance," Int. J. Contr., vol. 59, no. 2, pp. 439-461, 1994.

[16] B. R. Barmish, M. Corless, and G. Leitmann, "A new class of stabilizing controllers for uncertain dynamical systems," SIAM J. Contr. Optimizat., vol. 21 , pp. 246-255, 1983.

[17] M. Corless and G. Leitmann, "Continuous state feedback guaranteeing uniform ultimate boundedness of uncertain dynamic systems," IEEE Trans. Automatic Contr., vol. 26, pp. 1139-1144. 1981.

[18] F. Garofalo and G. Leitmann, "Guaranteeing ultimate boundedness and exponential rate of convergence for a class of nominally linear uncertain systems," ASME J. Dynamic Systems, Measurements, Contr., vol. 111, pp. $584-588,1989$

[19] D. Driankov, H. Hellendoorn, and M. Reinfrank, An Introduction to Fuzzy Control. Berlin, Germany: Springer-Verlag, 1993.

[20] M. Jamshidi, N. Vadiee, and T. J. Ress, Fuzzy Logic and Control. Englewood Cliffs, NJ: Prentice-Hall, 1993. 
[21] K. S. Narendra and A. M. Annaswamy, Stable Adaptive Systems, Prentice-Hall, 1989.

[22] R. M. Sanner and J. J. E. Slotine, "Gaussian networks for direct adaptive control," IEEE Trans. Neural Networks, vol. 3, pp. 837-864, June 1992.

[23] R. Ortega and M. W. Spong, "Adaptive motion control of rigid robotics: A tutorial," Automatica, vol. 25, pp. 877-888, 1989.

[24] M. W. Spong and M. Vidyasagar, Robot Dynamics and Control. New York: Wiley, 1989.

[25] L. X. Wang, Adaptive Fuzzy Systems and Control: Design and Stability Analysis. Englewood Cliffs, NJ: Prentice-Hall, 1994.

[26] B. S. Chen, C. H. Lee, and Y. C. Chang, " $H_{\infty}$ Tracking design of uncertain nonlinear SISO systems: Adaptive fuzzy approach," IEEE Trans. Fuzzy Syst., vol. 4, pp. 32-43, Feb. 1996.

[27] R. Isaacs, Differential Games. Huntington, NY: Kruger, 1975.

[28] Y. C. Chang and B. S. Chen, "A nonlinear adaptive $H_{\infty}$ tracking control design in robotic systems via neural networks," IEEE Trans. Contr. Syst. Technol., vol. 5, no. 1, pp. 13-29, Jan. 1997.

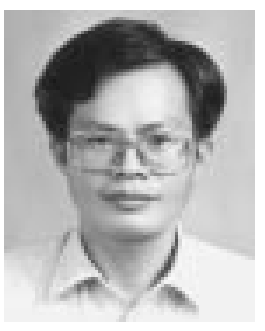

Bor-Sen Chen (SM'94) received the B.S. degree from Tatung Institute of Technology, the M.S. degree from National Central University, Taiwan, R.O.C., and the Ph.D. degree from the University of Southern California, Los Angeles, CA, in 1970, 1973, and 1982, respectively.

He was a Lecturer, Associate Professor, and Professor at Tatung Institute of Technology, from 1973-1987. He is currently a Professor at National Tsing-Hua University, Hsin-Chu, Taiwan, R.O.C. His current research interests are in control and signal processing.

Dr. Chen has received the Distinguished Research Award from the National Science Council of Taiwan four times. He is now also a Research Fellow of the National Science Council of Taiwan.

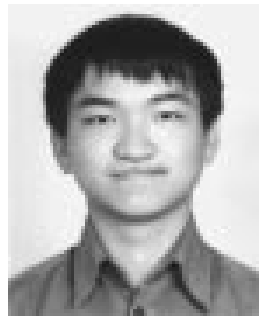

Huey-Jian Uang was born in Taichung, Taiwan, R.O.C. He received the B.S. degree from FengChia University, Taichung, Taiwan, and the M.S degree from National Tsing-Hua University, Hsin $\mathrm{Chu}$, Taiwan, in electrical engineering. He is currently working toward the Ph.D. degree in electrical engineering at National Tsing-Hua University.

His research interests include nonlinear control, adaptive systems, and robotics.

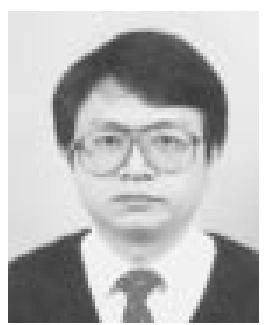

Chung-Shi Tseng received the B.S. degree from the Department of Electrical Engineering, National Cheng Kung University, Tainan, Taiwan, in 1984, and the M.S. degree from the Department of Electrical Engineering and Computer Engineering, University of New Mexico, Albuquerque, NM, in 1987. $\mathrm{He}$ is currently working toward the Ph.D. degree in the Department of Electrical Engineering, National Tsing-Hua University, Hsin Chu, Taiwan.

His research interests are in nonlinear control, adaptive systems, and robotics. 